\title{
MULTIDIMENSIONAL SCHRÖDINGER EQUATIONS WITH ABELIAN POTENTIALS
}

\author{
V. M. BUCHSTABER, J. C. EILBECK, V. Z. ENOLSKII, D. V. LEYKIN, AND \\ M. SALERNO
}

\begin{abstract}
We consider 2D and 3D complex Schrödinger equations with Abelian potentials and a fixed energy level. The potential, wave function, and the spectral Bloch variety are calculated in terms of the Kleinian hyperelliptic functions associated with a genus two hyperelliptic curve. In the special case in $2 \mathrm{D}$ when the curve covers two elliptic curves, exactly solvable Schrödinger equations are constructed in terms of the elliptic functions of these curves. The solutions obtained are illustrated by a number of plots.
\end{abstract}

\section{INTRODUCTION}

Modern physical technologies have led to the manufacture of new materials - films, super-lattices, 2D quantum dots arrays, etc., whose mathematical models are based on the 2D and 3D Schrödinger equation with periodic and quasiperiodic potentials. The recent discoveries of soliton theory, which involve the theory of Abelian functions, make it possible to construct exactly solvable Schrödinger equations with Abelian potentials. Progress in this area was made by Novikov and coworkers since the middle of the 1970s (see e.g. [19]). In [15], the multi-dimensional spectral problem for the Schrödinger equation under the action of an external magnetic field was considered, and the corresponding Bloch solutions were constructed at specific values of the energy. The case of a real pure potential was solved in Refs. [21] and [20] for some special hyperelliptic curves which permit involution with two stable points, leading to Prym varieties.

The complex theory of the 2D Schrödinger equation with an Abelian potential was developed by Buchstaber and Enolskii [11] by differentiating the addition theorem for the Baker function of genus two; in this approach, the 2D Schrödinger equation appeared as a compatibility condition for the ansatz introduced in [11]. The key ingredient of this technique is the use of the Weierstrass-Klein realization of the hyperelliptic functions, which is treated in the classical literature $[3,4]$ and more recently developed in [14]. 
The aim of the present paper is to develop the theory for $2 \mathrm{D}$ and 3D Schrödinger equations with an Abelian potential using, as the basic ingredient, the Baker function $\Phi(\boldsymbol{x} ; \boldsymbol{\alpha})$ of the hyperelliptic curve $V$ of genus $g$

$$
\Phi_{B}(\boldsymbol{x} ; \boldsymbol{\alpha})=\frac{\sigma(\boldsymbol{\alpha}-\boldsymbol{x})}{\sigma(\boldsymbol{\alpha}) \sigma(\boldsymbol{x})} \exp \left(\boldsymbol{\zeta}(\boldsymbol{\alpha})^{T} \boldsymbol{x}\right),
$$

where the variable $\boldsymbol{x}$ and spectral variable $\boldsymbol{\alpha}$ belong to the Jacobi varieties of the underlying hyperelliptic curve, the $\sigma$-function is a generalization of the Weierstrass $\sigma$-function to higher genera, and $\zeta_{i}=\partial / \partial x_{i} \ln \sigma(\boldsymbol{x}), i=1, \ldots, g$ are hyperelliptic $\zeta$-functions. We emphasize that the Baker function is defined on the product of Jacobians $\operatorname{Jac}(V) \times \operatorname{Jac}(V)$ in contrast to the Baker-Akhiezer function, which is defined on the product $V \times \operatorname{Jac}(V)$.

To explain the results, we first consider the $n$-dimensional Schrödinger equation

$$
\left\{\sum_{k=1}^{n} \frac{\partial^{2}}{\partial x_{k}^{2}}-\mathcal{U}(\boldsymbol{x})\right\} \Psi(\boldsymbol{x} ; \boldsymbol{\alpha})=\lambda(\boldsymbol{\alpha}) \Psi(\boldsymbol{x} ; \boldsymbol{\alpha}),
$$

where the potential $\mathcal{U}(\boldsymbol{x})$ depends on the column vector $\boldsymbol{x}$ belonging to the $n$-dimensional complex space $\mathbb{C}^{n}, \Psi(\boldsymbol{x} ; \boldsymbol{\alpha})$ is the wave function depending both on $\boldsymbol{x}$ and the spectral parameter, which is a column vector $\boldsymbol{\alpha} \in \mathbb{C}^{n}$, and $\lambda(\boldsymbol{\alpha})$ is the spectral function. We assume that the functions introduced have the following periodicity properties with respect to the $2 n n$-dimensional vectors, which are columns of the $n \times 2 n$ matrix $\left(2 \omega, 2 \omega^{\prime}\right)$, where $\omega, \omega^{\prime}$ are $n \times n$-matrices given by

$$
\begin{aligned}
& \mathcal{U}\left(\boldsymbol{x}+2 \omega \boldsymbol{n}+2 \omega^{\prime} \boldsymbol{m}\right)=\mathcal{U}(\boldsymbol{x}), \\
& \Psi\left(\boldsymbol{x}+2 \omega \boldsymbol{n}+2 \omega^{\prime} \boldsymbol{m} ; \boldsymbol{\alpha}\right)=\xi_{\boldsymbol{n}, \boldsymbol{m}}(\boldsymbol{\alpha}) \Psi(\boldsymbol{x} ; \boldsymbol{\alpha}), \\
& \Psi\left(\boldsymbol{x} ; \boldsymbol{\alpha}+2 \omega \boldsymbol{n}+2 \omega^{\prime} \boldsymbol{m}\right)=\Psi(\boldsymbol{x} ; \boldsymbol{\alpha}),
\end{aligned}
$$

where $\boldsymbol{n}, \boldsymbol{m}$ are arbitrary integer column vectors, and the function $\xi(\boldsymbol{\alpha})$ is the Bloch factor. The Bloch factor is assumed to be of the form

$$
\xi_{\boldsymbol{n}, \boldsymbol{m}}(\boldsymbol{\alpha})=\exp \left\{2 \boldsymbol{k}^{T}(\boldsymbol{\alpha})\left(\omega \boldsymbol{n}+\omega^{\prime} \boldsymbol{m}\right)\right\},
$$

and the quasi-momentum $\boldsymbol{k}(\boldsymbol{\alpha})$ is identified with the eigenvalue of the translation operator on the lattice.

The equations (1.3) and (1.5) represent a natural generalization of the usual Bloch theorem to the case of Abelian potentials. Indeed, after expressing the spectral parameter in terms of the quasi-momentum and considering translations in the crystal lattice by a vector of the form $2 \omega \boldsymbol{n}$, with $2 \omega=\left(2 \omega_{i, j}\right)_{i, j=1, \ldots, n}$ being the $n \times n$ period matrix, we have 
from $(1.3)$

$$
\Psi(\boldsymbol{x}+2 \omega \boldsymbol{n} ; \boldsymbol{k})=\mathrm{e}^{2 \boldsymbol{k}^{T} \omega \boldsymbol{n}} \Psi(\boldsymbol{x} ; \boldsymbol{k}) .
$$

Similarly, with respect to points of the reciprocal lattice, (1.4) gives

$$
\Psi(\boldsymbol{x} ; \boldsymbol{k}+2 \widetilde{\boldsymbol{\omega}} \boldsymbol{n})=\Psi(\boldsymbol{x} ; \boldsymbol{k}),
$$

where $2 \widetilde{\omega} \boldsymbol{n}$ is a reciprocal period, which is represented as a column of the $n \times n$ reciprocal period matrix $2 \widetilde{\omega}$, which will be shown later to be equal to $i \pi(\omega)^{-1}$.

These properties mean that the Schrödinger equation is considered on the product of tori $T \times T, T=\mathbb{C}^{n} / 2 \omega \oplus 2 \omega^{\prime}$, which we assume to be generated by a hyperelliptic curve $V$ and therefore $T$ is the Jacobian $\operatorname{Jac}(V)$ of the curve. Since the potential has the periodicity property (1.2), it is an Abelian function, as is the corresponding spectral function.

In this case, the periods $2 \omega$ and $2 \omega^{\prime}$ are generated by the holomorphic integrals over cycles of the curve $V$, and it is always possible to fix the parameters of the curve in such a way, that the period matrix $2 \omega$ is real and the period matrix $2 \omega^{\prime}$ is pure imaginary.

From the physical viewpoint, it is natural to consider the potential and the wave functions as functions of the argument $\imath \boldsymbol{x}+\Omega$, where $\boldsymbol{\Omega}$ is a real half period. Then the potential $\mathcal{U}(\imath \boldsymbol{x}+\Omega)$ is periodic with respect to the period lattice $2 \imath \omega^{\prime}$ and is smooth and real at real $\boldsymbol{x}$, and the wave function $\Psi\left(\imath \boldsymbol{x}+\boldsymbol{\Omega} ; \boldsymbol{\alpha}+\boldsymbol{\Omega}^{\prime}\right)$, where $\boldsymbol{\Omega}^{\prime}$ is a pure imaginary period, satisfy the Bloch property (1.6)

$$
\Psi\left(\imath \boldsymbol{x}+\boldsymbol{\Omega}+2 \omega^{\prime} \boldsymbol{n} ; \boldsymbol{k}(\boldsymbol{\alpha})\right)=\mathrm{e}^{2 \imath \boldsymbol{k}^{T}(\boldsymbol{\alpha}) \operatorname{Im}\left(\omega^{\prime}\right) \boldsymbol{n}} \Psi(\imath \boldsymbol{x}+\boldsymbol{\Omega} ; \boldsymbol{k}(\boldsymbol{\alpha})),
$$

with real quasi-momentum $\boldsymbol{k}(\boldsymbol{\alpha})$.

In this context, we shall call this periodicity property of the wave function the Bloch property. The subvariety $\mathcal{B} \subset \operatorname{Jac}(V)$ for which the spectral problem (1.1) can be solved is called the Bloch variety.

The Bloch variety, when parametrized in terms of the quasimomentum $\boldsymbol{k}$, and restricted to a fixed value of the energy $\mathcal{E}=\lambda(\boldsymbol{\alpha})=$ const, is called a Fermi variety, denoted by $\mathcal{F}$. If the Schrödinger equation permits solutions only at a fixed energy level, which is independent of the spectral parameter $\boldsymbol{\alpha}$, then the Bloch variety and the Fermi variety are isomorphic, but generally speaking $\operatorname{dim} \mathcal{B} \geq \operatorname{dim} \mathcal{F}$. The Fermi variety is a Fermi surface in the case $\operatorname{dim} \mathcal{F}=2$ (this situation is usually realized at $n=3$ ) and a Fermi curve in the case when $\operatorname{dim} \mathcal{F}=1$. In the theory of the electronic structure of metals, most interest lies in those exactly solvable Schrödinger equations which admit nontrivial Fermi varieties. 
In what follows we shall interpret all these quantities for the case of 2D and 3D Schrödingers equations in terms of Abelian functions of genus two and genus three hyperelliptic curves.

We would like to emphasise that the known methods of derivation of exact solutions of multidimensional Schrödingers equations suppose the separability of the potential, i.e. in $2 \mathrm{D}, \mathcal{U}(\boldsymbol{x})=\alpha \wp\left(x_{1}\right) \wp\left(x_{2}\right)$, where the $\wp\left(x_{i}\right)$ are the Weierstrass elliptic functions, and $\alpha=$ const. In this case the lattice structure is necessarily rectangular(cubic) in $2 \mathrm{D}(3 \mathrm{D})$. The non-separable potentials which we are considering enable us to investigate more general lattice symmetries, with a greater range of physical applications.

The paper is organized as follows. In Section 2 we consider the well known case of the Lamé equation in 1D elliptic potentials. In Section 3 we introduce a suitable generalization of the Kleinian function to higher genera and present a natural extension of the theory of the onegap Lamé potential to higher dimensions. The results of this section will not be restricted to two dimensions but will be valid also in the $3 \mathrm{D}$ case. In Section 4 we apply the general results of Section 3 to the $2 \mathrm{D}$ case, by deriving the explicit form of a certain family of Abelian potentials for which the 2D Schrödinger equation is exactly solvable. A set of figures for the potential profiles, showing that they are periodic, real and nonsingular, and therefore suitable for physical applications, is also given. The corresponding eigenvalues and eigenfunctions are also explicitly displayed. Section 5 is devoted to the case of reduction of the 2D Abelian potential and associated eigenfunction to elliptic functions. To do this we use the general results of Section 3 together with the explicit formulae for the deformation of the two-gap Lamé potential under the action of the KdV flow and the 3-particle dynamics over the locus, to derive exactly solvable 2D Schrödinger equations with elliptic potentials. Finally, in Section 6 we will consider the extension of the theory to the $3 \mathrm{D}$ case.

\section{The 1D Schrödinger Equation With ElLiptic Potentials}

One of the main problems in condensed matter physics is the construction of solutions of the Schrödinger equation with real and nonsingular periodic or quasi periodic potentials. Except in very few cases, this problem is in general not solvable without resorting to approximations.

One of the few cases for which exact solutions are known is provided by the remarkable example of the one dimensional Lamé equation with an elliptic potential (here and below we follow the standard notation 
of the theory of elliptic functions fixed in [7]),

$$
\begin{aligned}
& \text { (2.1) }\left\{\frac{\mathrm{d}^{2}}{\mathrm{~d} x^{2}}-\mathcal{U}(x)\right\} \Psi(x ; \alpha)=\wp(\alpha) \Psi(x ; \alpha), \\
& (2.2) \mathcal{U}(x)=2 \wp(x), \quad \Psi(x ; \alpha)=\Phi_{W}(x ; \alpha)=\frac{\sigma(\alpha-x)}{\sigma(\alpha) \sigma(x)} \exp \{\zeta(\alpha) x\} .
\end{aligned}
$$

Since in the following sections we will consider the generalization of this classical case to higher dimensions, we shall briefly review it here.

We recall that the Weierstrass functions $\wp, \zeta$ are given as logarithmic derivatives of the $\sigma$-function,

$$
\wp(x)=-\frac{\mathrm{d}^{2}}{\mathrm{~d} x^{2}} \ln \sigma(x), \quad \zeta(x)=\frac{\mathrm{d}}{\mathrm{d} x} \ln \sigma(x) .
$$

The $\sigma$-function, which is the generating function for the whole theory, is constructed from the elliptic curve

$$
w^{2}=4 z^{3}-g_{2} z-g_{3} \equiv 4\left(z-e_{1}\right)\left(z-e_{2}\right)\left(z-e_{3}\right) .
$$

equipped with a canonical basis of cycles $\mathfrak{a}, \mathfrak{b}$ as follows. The holomorphic differential and the associated meromorphic differential of the second kind are given respectively by $\mathrm{d} z / w$ and $z \mathrm{~d} z / w$. Their $\mathfrak{a}$ and $\mathfrak{b}$ periods

$$
\begin{aligned}
2 \omega & =\oint_{\mathfrak{a}} \frac{\mathrm{d} z}{w}, \quad 2 \omega^{\prime}=\oint_{\mathfrak{b}} \frac{\mathrm{d} z}{w} \\
2 \eta & =-\oint_{\mathfrak{a}} \frac{z \mathrm{~d} z}{w}, \quad 2 \eta^{\prime}=-\oint_{\mathfrak{b}} \frac{z \mathrm{~d} z}{w}
\end{aligned}
$$

satisfy the Legendre relation

$$
\eta \omega^{\prime}-\omega \eta^{\prime}=\frac{\imath \pi}{2}
$$

Then the Weierstrass $\sigma$-function has the form

$$
\sigma(x)=\sqrt{\frac{\pi}{\omega}} \frac{1}{\sqrt[8]{\Delta}} \exp \left(\frac{\eta x^{2}}{2 \omega}\right) \vartheta_{1}\left(\frac{x}{2 \omega} \mid \tau\right),
$$

where $\vartheta_{1}$ is the Jacobian $\theta$-function, $\tau=\omega^{\prime} / \omega$ and $\Delta=16\left(e_{2}-e_{3}\right)^{2}\left(e_{3}-\right.$ $\left.e_{1}\right)^{2}\left(e_{1}-e_{2}\right)^{2}$. This $\sigma$-function can be also be represented as the power series

$$
\sigma(x)=x-\frac{g_{2} x^{5}}{2^{4} \cdot 3 \cdot 5}+\frac{g_{3} x^{7}}{2^{3} \cdot 3 \cdot 5 \cdot 7}+\ldots
$$


with coefficients connected by a recursion relations first found by Weierstrass [22] and documented in [1] (see also [17]). The Weierstrass $\sigma$ function has the periodicity property

$$
\begin{aligned}
& \sigma(x+2 n \omega+2 m \omega) \\
& =(-1)^{n+m+m n} \exp \left\{2\left(n \eta+m \eta^{\prime}\right)\left(x+n \omega+m \omega^{\prime}\right)\right\} \sigma(x),
\end{aligned}
$$

which leads to the following expression for the Bloch factor

$$
\xi_{i}(\alpha)=\exp \left(2 \omega_{i} \zeta(\alpha)-2 \alpha \eta_{i}\right), \quad i=1,2,3 .
$$

The functions $\xi_{i}(\alpha)$ are elliptic functions with periods $2 \omega, 2 \omega^{\prime}$, because of the Legendre relation.

To give the physical interpretation of the above formulae we shall fix the potential and the wave function as follows

$$
\mathcal{U}(x)=2 \wp(\imath x+\omega), \quad \Psi(x ; \alpha)=\Phi_{W}\left(\imath x+\omega ; \alpha+\omega^{\prime}\right), \quad x, \alpha \in \mathbb{R}
$$

which has the real period $-2 \imath \omega^{\prime}$; the associated quasi-momentum is given by the formula

$$
k(\alpha)=\zeta\left(\alpha+\omega^{\prime}\right)-\frac{\eta^{\prime}}{\omega^{\prime}}\left(\alpha+\omega^{\prime}\right), \quad \alpha \in \mathbb{R},
$$

The wave function, considered as a function of $k$ instead of $\alpha$, has the periodicity property (1.7) with the reciprocal period

$$
2 \widetilde{\omega}=\frac{\imath \pi}{\omega^{\prime}} .
$$

The Bloch variety $\mathcal{B}$ is the Jacobian of the elliptic curve, which is isomorphic in this case to the elliptic curve itself.

We remark here that the 1-dimensional finite-gap potentials appear to be important in applications. For example Belokolos proved in 1980 [8] that the exact solution of the famous Peierls problem is a finite-gap potential.

The simplest generalization of (2.1) to the $n$-dimensional case can be obtained if we consider the separable potential

$$
\mathcal{U}(\boldsymbol{x})=2 \sum_{k=1}^{n} \wp\left(\imath x_{k}+\omega^{(k)} ; 2 \omega^{(k)}, 2 \omega^{(k)^{\prime}}\right),
$$

where $\wp\left(\cdot ; 2 \omega^{(k)}, 2 \omega^{(k)^{\prime}}\right)$ are Weierstrass elliptic functions with periods $2 \omega^{(k)}$ and $2 \omega^{(k)^{\prime}}$. The energy $\mathcal{E}$ and components of the quasi-momenta 
$\boldsymbol{k}$ are then

$$
\begin{aligned}
\mathcal{E} & =\sum_{k=1}^{n} \wp\left(\alpha_{k}+\omega^{(k)^{\prime}} ; 2 \omega^{(k)}, 2 \omega^{(k)^{\prime}}\right), \\
k_{j}\left(\alpha_{j}\right) & =\zeta\left(\alpha_{j}\right)-\frac{\eta^{(j)^{\prime}}}{\omega^{(j)^{\prime}}} \alpha_{j}, \quad j=1, \ldots, n .
\end{aligned}
$$

The wave function is given in this case as a product of the functions $\Phi_{W}$.

In the case $n=3$ such potentials lead to non-trivial and sometimes very interesting Fermi surfaces. For example, Baryakhtar et al. [6] have used separable potentials to calculate successfully the electron energy of metals and high-temperature superconductors, and Belokolos and Korostil [5] have studied the electron-phonon interaction function.

Moreover, a general theory for the Schrödinger equations with separable multidimensional Lamé potentials with an arbitrary number of gaps in the spectrum was recently developed in [10]. This approach was shown to be effective for the exact computation of the energy bands and Fermi surfaces of 2D lattices with square or rectangular symmetry. The extension of these results, however, to lattices with more general spatial symmetries seems problematic in the context of separable multidimensional Lamé potentials.

We shall develop below another generalization, which leads to a nonseparable potential. Namely we shall show that the Lamé equation (2.1) can be generalized to higher dimensions within the WeierstrassKlein generalization of Weierstrass elliptic function theory to higher genera, following $[3,4]$ and also $[13,14]$. This generalization enables us to treat more general symmetries than the separable case.

\section{Multidimensional Schrödinger equations aS GENERALIZED LAMÉ EQUATIONS OF HIGHER GENERA}

To develop the theory of the multidimensional Schrödinger equation we need a suitable generalization of the fundamental $\sigma$-function of the hyperelliptic curve $V=V(w, z)$ of genus $g$

$$
w^{2}=4 \prod_{i=1}^{2 g+1}\left(z-e_{i}\right) \equiv 4 z^{2 g+1}+\sum_{i=0}^{2 g} \lambda_{i} z^{i}
$$

by the following formula, which is analogous to that in the elliptic case

$$
\sigma(\boldsymbol{x})=\sqrt{\frac{\pi^{g}}{\operatorname{det} 2 \omega}} \frac{1}{\sqrt[4]{\prod_{i \neq j}\left(e_{i}-e_{j}\right)}} \exp \left\{\boldsymbol{x}^{T} \varkappa \boldsymbol{x}\right\} \theta[\varepsilon]\left((2 \omega)^{-1} \boldsymbol{x} \mid \tau\right),
$$


where

$$
[\varepsilon]=\left[\begin{array}{c}
\boldsymbol{\varepsilon}^{\prime T} \\
\boldsymbol{\varepsilon}^{T}
\end{array}\right]=\left[\begin{array}{lll}
\varepsilon_{1}^{\prime} & \ldots & \varepsilon_{g}^{\prime} \\
\varepsilon_{1} & \ldots & \varepsilon_{g}
\end{array}\right]
$$

is the necessarily half integer characteristic of the vector of Riemann constants, and $\theta[\varepsilon](\boldsymbol{v} \mid \tau)$ is the standard multi-dimensional theta function with characteristic

$$
\theta[\varepsilon](\boldsymbol{v} \mid \tau)=\sum_{\boldsymbol{m} \in \mathbb{Z}^{g}} \exp \left\{\pi \imath\left(\boldsymbol{m}+\boldsymbol{\varepsilon}^{\prime}\right)^{T} \tau\left(\boldsymbol{m}+\boldsymbol{\varepsilon}^{\prime}\right)+2 \pi \imath\left(\boldsymbol{m}+\boldsymbol{\varepsilon}^{\prime}\right)^{T}(\boldsymbol{v}+\boldsymbol{\varepsilon})\right\} .
$$

The matrix

$$
\varkappa=\eta(2 \omega)^{-1}
$$

is the symmetric matrix which generalizes the factor $\eta / 2 \omega$ in the exponential (2.7) to the higher genera. The $2 g \times 2 g$ period matrix, $\left(\begin{array}{cc}\omega & \omega^{\prime} \\ \eta & \eta^{\prime}\end{array}\right)$ satisfies the generalized Legendre relation

$$
\left(\begin{array}{cc}
\omega & \omega^{\prime} \\
\eta & \eta^{\prime}
\end{array}\right)\left(\begin{array}{cc}
0 & -1_{g} \\
1_{g} & 0
\end{array}\right)\left(\begin{array}{cc}
\omega & \omega^{\prime} \\
\eta & \eta^{\prime}
\end{array}\right)^{T}\left(\begin{array}{cc}
0 & -1_{g} \\
1_{g} & 0
\end{array}\right)=-\frac{\imath \pi}{2},
$$

where the $g \times g$ period matrices $2 \omega, 2 \omega^{\prime}, 2 \eta, 2 \eta^{\prime}$ are

$$
\begin{aligned}
2 \omega & =\left(\oint_{\mathfrak{a}_{i}} \mathrm{~d} u_{j}\right)_{i, j=1, \ldots, g}, \quad 2 \omega^{\prime}=\left(\oint_{\mathfrak{b}_{i}} \mathrm{~d} u_{j}\right)_{i, j=1, \ldots, g}, \\
2 \eta & =\left(-\oint_{\mathfrak{a}_{i}} \mathrm{~d} r_{j}\right)_{i, j=1, \ldots, g}, 2 \eta^{\prime}=\left(-\oint_{\mathfrak{b}_{i}} \mathrm{~d} r_{j}\right)_{i, j=1, \ldots, g} .
\end{aligned}
$$

Here the $\mathrm{d} u_{i}$ are the holomorphic differentials

$$
\mathrm{d} \mathbf{u}^{T}=\left(\mathrm{d} u_{1}, \ldots, \mathrm{d} u_{g}\right), \quad \mathrm{d} u_{k}=\frac{z^{k-1} \mathrm{~d} z}{w},
$$

and the $\mathrm{d} r_{i}$ are the differentials of the second kind with a pole at infinity

$$
\begin{aligned}
& \mathrm{d} \mathbf{r}^{T}=\left(\mathrm{d} r_{1}, \ldots, \mathrm{d} r_{g}\right), \\
& \mathrm{d} r_{j}=\sum_{k=j}^{2 g+1-j}(k+1-j) \lambda_{k+1+j} \frac{z^{k} \mathrm{~d} z}{4 w}, \quad j=1, \ldots, g .
\end{aligned}
$$

The Kleinian $\sigma$-function has the following periodicity property

$$
\begin{aligned}
\sigma(\boldsymbol{x}+2 \boldsymbol{\Omega}(\boldsymbol{n}, \boldsymbol{m}))= & \exp \left\{2 \boldsymbol{E}^{T}(\boldsymbol{n}, \boldsymbol{m})(\boldsymbol{x}+\boldsymbol{\Omega}(\boldsymbol{n}, \boldsymbol{m}))\right\} \\
& \times \exp \left\{-\imath \pi \boldsymbol{n}^{T} \boldsymbol{m}-2 \imath \pi \boldsymbol{\varepsilon}^{T} \boldsymbol{m}\right\} \sigma(\boldsymbol{x}),
\end{aligned}
$$

where $\boldsymbol{E}(\boldsymbol{n}, \boldsymbol{m})=\eta \boldsymbol{n}+\eta^{\prime} \boldsymbol{m}, \boldsymbol{\Omega}(\boldsymbol{n}, \boldsymbol{m})=\omega \boldsymbol{n}+\omega^{\prime} \boldsymbol{m}, \boldsymbol{n}, \boldsymbol{m} \in \mathbb{Z}^{g}$ and $\varepsilon^{T}$ is the lower line of the characteristic of the vector of the Riemann 
constant. The Kleinian $\zeta$ and $\wp$-functions are introduced through logarithmic derivatives of the Kleinian $\sigma$-function,

$$
\begin{aligned}
\zeta_{i}(\boldsymbol{x}) & =\frac{\partial \ln \sigma(\boldsymbol{x})}{\partial x_{i}}, \quad \wp_{i, j}(\boldsymbol{x})=-\frac{\partial^{2} \ln \sigma(\boldsymbol{x})}{\partial x_{i} \partial x_{j}}, \\
\wp_{i, j, k}(\boldsymbol{x}) & =-\frac{\partial^{3} \ln \sigma(\boldsymbol{x})}{\partial x_{i} \partial x_{j} \partial x_{k}}, \quad i, j, k=1, \ldots, g \quad \text { etc. }
\end{aligned}
$$

We will omit commas between indices when the resulting formula is unambiguous. The Abel map $\mathfrak{A}:(V)^{g} \rightarrow \operatorname{Jac}(V)$ of the symmetrised product $V \times \cdots \times V$ to the Jacobi variety $\operatorname{Jac}(V)=\mathbb{C}^{g} / 2 \omega \oplus 2 \omega^{\prime}$ of the curve $V$ is defined by the equations

$$
\sum_{k=1}^{g} \int_{\left(0, e_{k}\right)}^{\left(w_{k}, z_{k}\right)} \mathrm{d} \mathbf{u}=\boldsymbol{x} .
$$

The Kummer variety is defined as the factor $\operatorname{Kum}(V)=\operatorname{Jac}(V) / \boldsymbol{x} \rightarrow$ $-\boldsymbol{x}$ by the involution $\boldsymbol{x} \longrightarrow-\boldsymbol{x}$.

The principal results of the theory of the hyperelliptic Kleinian can be formulated using the $(g+2) \times(g+2)$-matrix

$$
\begin{array}{r}
H=\left\{h_{i, k}\right\}_{i, k=1, \ldots, g+2}, \quad h_{i k}=4 \wp_{i-1, k-1}-2 \wp_{k, i-2}-2 \wp_{i, k-2} \\
+\frac{1}{2}\left(\delta_{i, k}\left(\lambda_{2 i-2}+\lambda_{2 k-2}\right)+\delta_{k, i+1} \lambda_{2 i-1}+\delta_{i, k+1} \lambda_{2 k-1}\right) .
\end{array}
$$

We denote the minors of the matrix $H$ as follows

$$
H\left[\begin{array}{c}
k_{1} \ldots k_{n} \\
j_{1} \ldots j_{m}
\end{array}\right]\left\{h_{i_{k}, j_{l}}\right\}_{k=1, \ldots, m ; l=1, \ldots, n} .
$$

Theorem 3.1 ([14]). The matrix $H$ has the following properties

- Let $\left(w_{1}, z_{1}\right), \ldots,\left(w_{g}, z_{g}\right)$ be an divisor and $\boldsymbol{Z}=\left(1, z, \ldots, z^{g+1}\right)$, then for arbitrary vectors $w_{r} w_{s}=\boldsymbol{Z}_{r}^{T} H \boldsymbol{Z}_{s}, \quad$ and in particular $\boldsymbol{Z}^{T} H \boldsymbol{Z}=\sum_{i=0}^{2 g+2} \lambda_{i} z^{i}$.

- Let $\left(w_{1}, z_{1}\right), \ldots,\left(w_{g}, z_{g}\right)$ be the divisor, then the vectors $\boldsymbol{Z}=$ $\left(1, z_{r}, \ldots, z_{r}^{g+1}\right), r=1, \ldots, g$ are orthogonal to the last column of the matrix $H$ or equivalently the $z_{r}$ are the roots of the equation

$$
z^{g}-\wp_{g g}(\boldsymbol{u}) z^{g-1}-\ldots-\wp_{1 g}(\boldsymbol{u})=0,
$$

which yields the solution of the Jacobi inversion problem, where the second coordinate of the divisor is defined as follows

$$
w_{k}=-\sum_{j=1}^{g} \wp_{j g g}(\boldsymbol{u}) z_{k}^{g-j} .
$$


- $\operatorname{rank} H=3$ at generic points and

$$
-\frac{1}{4} \wp_{i g g} \wp_{k g g}=\operatorname{det} H\left[\begin{array}{l}
k g+1 g+2 \\
i g+1 g+2
\end{array}\right], \quad \forall i, k=1, \ldots, g .
$$

The intersection of the $g(g+1) / 2$ cubics defines the Jacobi variety as an algebraic variety in $\mathbb{C}^{g+\frac{1}{2} g(g+1)}$.

- The intersection of the $g(g-1) / 2$ quartics

$$
\operatorname{det} H\left[\begin{array}{c}
k l g+1 g+2 \\
i j g+1 g+2
\end{array}\right]=0, \forall i \neq j, k \neq l=1, \ldots, g
$$

defines the Kummer variety as an algebraic variety in $\mathbb{C}^{\frac{1}{2} g(g+1)}$.

- The following equality is valid

$$
\mathbf{R}^{T} \boldsymbol{\pi}_{j l} \boldsymbol{\pi}_{i k}^{T} \mathbf{S}=\frac{1}{4} \operatorname{det}\left(\begin{array}{cc}
H\left[\begin{array}{cc}
i k g+1 g+2 \\
j l g+1 g+2
\end{array}\right] & \mathbf{S} \\
\mathbf{R}^{T} & 0
\end{array}\right)
$$

where $\mathbf{R}, \mathbf{S} \in \mathbb{C}^{4}$ are arbitrary vectors and

$$
\boldsymbol{\pi}_{i k}=\left(\begin{array}{c}
-\wp_{g g k} \\
\wp_{g g i} \\
\wp_{g, i, k-1}-\wp_{g, i-1, k} \\
\wp_{g-1, i, k-1}-\wp_{g-1, k, i-1}+\wp_{g, k, i-2}-\wp_{g, i, k-2}
\end{array}\right) .
$$

On the basis of the above relations, we shall construct the linear differential operators for which the spectral variety will be defined in $\operatorname{Jac}(V)$. Following Baker (see [3, page 421]) we define a function on $\operatorname{Jac}(V) \times \operatorname{Jac}(V)$.

Definition 3.1. The standard Baker function $\Phi_{B}$ of the curve $V$ is the function on the product $\operatorname{Jac}(V) \times \operatorname{Jac}(V)$, and is defined as follows

$$
\begin{gathered}
\Phi_{B}: \operatorname{Jac}(V) \times \operatorname{Jac} V \rightarrow \mathbb{C} \\
\Phi_{B}(\boldsymbol{x} ; \boldsymbol{\alpha})=\frac{\sigma(\boldsymbol{\alpha}-\boldsymbol{x})}{\sigma(\boldsymbol{\alpha}) \sigma(\boldsymbol{x})} \exp \left(\boldsymbol{\zeta}^{T}(\boldsymbol{\alpha}) \boldsymbol{x}\right),
\end{gathered}
$$

where $\boldsymbol{\zeta}^{T}(\boldsymbol{\alpha})=\left(\zeta_{1}(\boldsymbol{\alpha}), \ldots, \zeta_{g}(\boldsymbol{\alpha})\right)$, and

$$
\boldsymbol{x}=\sum_{k=1}^{g} \int_{\left(0, e_{k}\right)}^{\left(w_{k}, z_{k}\right)} \mathrm{d} \mathbf{u}, \quad \boldsymbol{\alpha}=\sum_{k=1}^{g} \int_{\left(0, e_{k}\right)}^{\left(\nu_{k}, \mu_{k}\right)} \mathrm{d} \mathbf{u} \in \operatorname{Jac}(V),
$$

where $\left(\left(w_{1}, z_{1}\right), \ldots,\left(w_{g}, z_{g}\right)\right)$ and $\left(\left(\nu_{1}, \mu_{1}\right), \ldots,\left(\nu_{g}, \mu_{g}\right)\right)$ are nonspecial divisors on $V$. The $\Phi_{B}$-function is meromorphic in $\boldsymbol{x}$ and has the periodicity property (1.3) with the Bloch factor

$$
\xi_{\boldsymbol{n}, \boldsymbol{m}}(\alpha)=\exp \left\{2 \boldsymbol{\zeta}^{T}(\boldsymbol{\alpha}) \boldsymbol{\Omega}(\boldsymbol{n}, \boldsymbol{m})-2 \boldsymbol{E}^{T}(\boldsymbol{n}, \boldsymbol{m}) \boldsymbol{\alpha}\right\}, \quad i=1, \ldots, g,
$$

where $\boldsymbol{\Omega}(\boldsymbol{n}, \boldsymbol{m})=\omega \boldsymbol{n}+\omega^{\prime} \boldsymbol{m}, \quad \boldsymbol{E}(\boldsymbol{n}, \boldsymbol{m})=\eta \boldsymbol{n}+\eta^{\prime} \boldsymbol{m}, \quad \boldsymbol{n}, \boldsymbol{m} \in \mathbb{Z}^{g}$ are arbitrary integer vectors. We shall call the Baker function any function, which is meromorphic on $\operatorname{Jac}(V) \times \operatorname{Jac}(V)$ and has the periodicity 
property (1.3) with the Bloch factors (3.16). Evidently in the case of genus one $\Phi_{W} \equiv \Phi_{B}$.

We remark, that the spectral variety of the standard Baker function is the Jacobi variety of the curve, in contrast with the Baker-Akhiezer function, whose spectral parameter is evaluated on the curve. In our notation, the Baker-Akhiezer function is $\Phi_{B A}(\boldsymbol{u} ;(\mu, \nu))$, which is given by the formula (see [18])

$$
\Phi_{B A}(\boldsymbol{x} ;(\mu, \nu))=\frac{\sigma\left(\int_{\left(\nu_{0}, \mu_{0}\right)}^{(\nu, \mu)} \mathrm{d} \boldsymbol{u}-\boldsymbol{x}\right)}{\sigma(\boldsymbol{x})} \exp \left(\int_{\left(\nu_{0}, \mu_{0}\right)}^{(\nu, \mu)} \mathrm{d} \mathbf{r}^{T} \boldsymbol{x}\right),
$$

where $(\nu, \mu) \in V$, and $\boldsymbol{x} \in \operatorname{Jac}(V)$. The function $\Phi_{B A}$ solves the one dimensional Schrödinger equation with the potential $2 \wp_{g g}$

$$
\left(\partial_{g}^{2}-2 \wp_{g g}\right) \Phi_{B A}=\left(z+\frac{\lambda_{2 g}}{4}\right) \Phi_{B A},
$$

with respect to $u_{g}$ for all $(\nu, \mu) \in V$.

Let us fix as the period lattice the matrix of real periods $2 \omega$. In analogy with the case of genus one, the Bloch factor (3.16) of the standard Baker function can be written as

$$
\begin{aligned}
\xi_{\boldsymbol{n}}(\boldsymbol{\alpha}) & =\exp \left\{2 \boldsymbol{k}^{T}(\boldsymbol{\alpha}) \omega \boldsymbol{n}\right\} \\
\boldsymbol{k}(\boldsymbol{\alpha}) & =\boldsymbol{\zeta}\left(\boldsymbol{\alpha}+\boldsymbol{\Omega}^{\prime}\right)-2 \varkappa\left(\boldsymbol{\alpha}+\boldsymbol{\Omega}^{\prime}\right),
\end{aligned}
$$

where $\Omega^{\prime}$ is an imaginary half period.

To prove the last formula we take into account the definition and the symmetry property of the matrix $\varkappa$. We have

$$
(\eta \boldsymbol{n})^{T} \boldsymbol{\beta}=(\varkappa(2 \omega) \boldsymbol{n})^{T} \boldsymbol{\beta}=2 \boldsymbol{\beta}^{T} \varkappa \omega \boldsymbol{n} .
$$

The Bloch factor is an Abelian function, whose periodicity properties are provided by the periodicity property of the $\zeta$-function and the generalized Legendre relation (3.5).

We shall prove the following

Proposition 3.2. Let $V$ be a hyperelliptic curve of genus $g<4$. Then the $g+1+\frac{1}{2} g(g+1)$ Baker functions on $\operatorname{Jac}(V) \times \operatorname{Jac}(V)$ are

$$
\begin{aligned}
F_{0}(\boldsymbol{x} ; \boldsymbol{\alpha}) & =\Phi_{B}(\boldsymbol{x} ; \boldsymbol{\alpha}), \\
(3.19) F_{i}(\boldsymbol{x} ; \boldsymbol{\alpha}) & =\frac{\partial}{\partial x_{i}} \Phi_{B}(\boldsymbol{x} ; \boldsymbol{\alpha}), \quad i=1, \ldots, g, \\
F_{i j}(\boldsymbol{x} ; \boldsymbol{\alpha}) & =\left\{\frac{\partial^{2}}{\partial x_{i} \partial x_{j}}-2 \wp_{i j}(\boldsymbol{x})\right\} \Phi_{B}(\boldsymbol{x} ; \boldsymbol{\alpha}), \quad i \leq j=1, \ldots, g,
\end{aligned}
$$


where $\Phi_{B}(\boldsymbol{x} ; \boldsymbol{\alpha})$ is the standard Baker function of the curve. These functions, regarded as functions of $\boldsymbol{x}$, are linearly dependent, i.e. there exists at least one relation between them of the form

$$
c_{0}(\boldsymbol{\alpha}) F_{0}(\boldsymbol{x} ; \boldsymbol{\alpha})+\sum_{i=1}^{g} c_{i}(\boldsymbol{\alpha}) F_{i}(\boldsymbol{x} ; \boldsymbol{\alpha})+\sum_{i \leq j=1, \ldots g} c_{i j}(\boldsymbol{\alpha}) F_{i j}(\boldsymbol{x} ; \boldsymbol{\alpha})=0,
$$

where not all functions in $\boldsymbol{\alpha}: c_{0}(\boldsymbol{\alpha}), c_{i}(\boldsymbol{\alpha}), c_{i j}(\boldsymbol{\alpha})$ are equal to zero.

Proof. We shall prove the statement for $g=2$ and $g=3$. Let us write the standard addition theorem of the second order $\theta$-functions

$$
\begin{aligned}
& \theta\left[\begin{array}{c}
\boldsymbol{\varepsilon}^{\prime T} \\
\boldsymbol{\varepsilon}^{T}+\boldsymbol{\gamma}^{T}
\end{array}\right](\boldsymbol{v}+\boldsymbol{u} \mid \tau) \theta\left[\begin{array}{c}
\boldsymbol{\varepsilon}^{\prime T} \\
\boldsymbol{\varepsilon}^{T}
\end{array}\right](\boldsymbol{v}-\boldsymbol{u} \mid \tau) \\
& \quad=\sum_{2 \boldsymbol{\delta} \in(\mathbb{Z} / 2 \mathbb{Z})^{g}}(-1)^{4 \boldsymbol{\varepsilon}^{T} \boldsymbol{\delta}} \theta\left[\begin{array}{c}
\boldsymbol{\delta}^{T} \\
\boldsymbol{\gamma}^{T}
\end{array}\right](2 \boldsymbol{v} \mid 2 \tau) \theta\left[\begin{array}{c}
\boldsymbol{\varepsilon}^{\prime T}+\boldsymbol{\delta}^{T} \\
\boldsymbol{\gamma}^{T}
\end{array}\right](2 \boldsymbol{u} \mid 2 \tau),
\end{aligned}
$$

Now set $\boldsymbol{u}=0$ and $\boldsymbol{\gamma}=\mathbf{0}$,

(3.21)

$$
\theta^{2}\left[\begin{array}{c}
\boldsymbol{\varepsilon}^{\prime T} \\
\boldsymbol{\varepsilon}^{T}
\end{array}\right](\boldsymbol{v} \mid \tau)=\sum_{2 \boldsymbol{\delta} \in(\mathbb{Z} / 2 \mathbb{Z})^{g}}(-1)^{4 \boldsymbol{\varepsilon}^{T} \boldsymbol{\delta}^{T}} \theta\left[\begin{array}{l}
\boldsymbol{\delta}^{T} \\
\mathbf{0}^{T}
\end{array}\right](2 \boldsymbol{v} \mid 2 \tau) \theta\left[\begin{array}{c}
\boldsymbol{\varepsilon}^{T}+\boldsymbol{\delta}^{T} \\
\mathbf{0}^{T}
\end{array}\right](\mathbf{0} \mid 2 \tau),
$$

where $\boldsymbol{\delta}^{T}=\left(\delta_{1}, \ldots, \delta_{g}\right)$ with $2 \delta_{i}=1$ or $0, i=1, \ldots g$. We multiply both sides of (3.21) by the factor $\exp \left\{2 \boldsymbol{u}^{T} \boldsymbol{\varkappa} \boldsymbol{u}\right\}$, where $\boldsymbol{v}=(2 \omega)^{-1} \boldsymbol{u}$ and the matrix $\varkappa$ is given in the definition of the fundamental $\sigma$-function. One can see that the entire functions

$$
\begin{aligned}
\psi\left[\begin{array}{c}
\boldsymbol{\varepsilon}^{\prime} \\
\boldsymbol{\varepsilon}^{T}
\end{array}\right](\boldsymbol{v} \mid \tau) & =\exp \left\{2 \boldsymbol{u}^{T} \varkappa \boldsymbol{u}\right\} \theta^{2}\left[\begin{array}{c}
\boldsymbol{\varepsilon}^{\prime T} \\
\boldsymbol{\varepsilon}^{T}
\end{array}\right](\boldsymbol{v} \mid \tau), \\
\widetilde{\psi}\left[\begin{array}{l}
\boldsymbol{\delta}^{T} \\
\mathbf{0}^{T}
\end{array}\right](2 \boldsymbol{v} \mid 2 \tau) & =\exp \left\{2 \boldsymbol{u}^{T} \varkappa \boldsymbol{u}\right\} \theta\left[\begin{array}{l}
\boldsymbol{\delta}^{T} \\
\mathbf{0}^{T}
\end{array}\right](2 \boldsymbol{v} \mid 2 \tau) .
\end{aligned}
$$

have the same periodicity property

$$
\begin{aligned}
& \psi\left[\begin{array}{l}
\boldsymbol{\delta}^{T} \\
\mathbf{0}^{T}
\end{array}\right]\left(2 \boldsymbol{v}+2 \boldsymbol{\Omega}\left(\boldsymbol{m}, \boldsymbol{m}^{\prime}\right) \mid \tau\right) \\
& \quad=\exp \left\{2 \boldsymbol{E}^{T}\left(\boldsymbol{m}, \boldsymbol{m}^{\prime}\right)\left(\boldsymbol{x}+\boldsymbol{\Omega}\left(\boldsymbol{m}, \boldsymbol{m}^{\prime}\right)\right)\right\} \psi\left[\begin{array}{l}
\boldsymbol{\delta}^{T} \\
\mathbf{0}^{T}
\end{array}\right](2 \boldsymbol{v} \mid \tau),
\end{aligned}
$$

with $\boldsymbol{E}\left(\boldsymbol{m}, \boldsymbol{m}^{\prime}\right)=\eta \boldsymbol{m}+\eta^{\prime} \boldsymbol{m}^{\prime}$ as defined above. Therefore any $2^{g}+1$ entire functions which posses the periodicity property (3.23) are linearly dependent. Moreover this statement can be extended to the case of 
entire functions which have the periodicity property

$$
\begin{aligned}
& \psi\left(\boldsymbol{v}+2 \boldsymbol{\Omega}\left(\boldsymbol{m}, \boldsymbol{m}^{\prime}\right)\right) \\
& \quad=\exp \left\{2 \boldsymbol{E}^{T}\left(\boldsymbol{m}, \boldsymbol{m}^{\prime}\right)\left(\boldsymbol{x}+\boldsymbol{\Omega}\left(\boldsymbol{m}, \boldsymbol{m}^{\prime}\right)\right)+\gamma\left(\boldsymbol{m}, \boldsymbol{m}^{\prime}\right)\right\} \psi(\boldsymbol{v}),
\end{aligned}
$$

where $\gamma\left(\boldsymbol{m}, \boldsymbol{m}^{\prime}\right)$ is some constant. The proof given is valid for genera satisfying the inequality

$$
2^{g}<g+\frac{g(g+1)}{2} .
$$

These are the cases $g=2$ and $g=3$.

We remark, that in the case of $g=2$, the statement of Proposition 3.2 was derived in [11], see also [13], as the condition of the validity of the addition theorem for the Baker function

$$
\boldsymbol{\Phi}_{B}(\boldsymbol{u}+\boldsymbol{v} ; \boldsymbol{\alpha})=\frac{\boldsymbol{Y}^{T}(\boldsymbol{u} ; \boldsymbol{\alpha}) A \boldsymbol{Y}(\boldsymbol{v} ; \boldsymbol{\alpha})}{\boldsymbol{X}^{T}(\boldsymbol{u}) A \boldsymbol{X}(\boldsymbol{v})}
$$

with the integer $4 \times 4$-matrix $A$, where $\boldsymbol{X}(\boldsymbol{u})$ is a 4 component meromorphic vector function and the 4 component vector function $\boldsymbol{Y}(\boldsymbol{u} ; \boldsymbol{\alpha})$ has the periodicity properties $(1.3,1.4)$.

The origin of the ansatz (3.25) is explained as follows. In the case $g=1,(3.25)$ follows from the Weierstrass addition formula for the $\sigma$-functions,

$$
\frac{\sigma(u+v) \sigma(u-v)}{\sigma^{2}(u) \sigma^{2}(v)}=\wp(v)-\wp(u),
$$

which can be written in the equivalent form

$$
\Phi_{W}(u+v ; \alpha)=\frac{\Phi_{W}(u ; \alpha) \Phi_{W}^{\prime}(v ; \alpha)-\Phi_{W}(v ; \alpha) \Phi_{W}^{\prime}(u ; \alpha)}{\wp(u)-\wp(v)} .
$$

This last equality can be rewritten in the form (3.25) with the vectors

$$
\boldsymbol{Y}(u ; \alpha)=\left(\begin{array}{c}
\Phi(u ; \alpha) \\
\Phi^{\prime}(u ; \alpha)
\end{array}\right), \quad \boldsymbol{X}(u)=\left(\begin{array}{c}
\wp(u) \\
1
\end{array}\right)
$$

and the $2 \times 2$-matrix

$$
A=\left(\begin{array}{cc}
0 & -1 \\
1 & 0
\end{array}\right)
$$

In the case of genus $g=2$, a solution of (3.25) was found in [11] with

$$
\begin{aligned}
& \boldsymbol{X}^{T}(\boldsymbol{u})=\left(\wp_{22}(\boldsymbol{u}), \wp_{12}(\boldsymbol{u}), \wp_{11}(\boldsymbol{u}), 1\right), \\
& \boldsymbol{Y}^{T}(\boldsymbol{u} ; \boldsymbol{\alpha})=C\left(F_{0}(\boldsymbol{u} ; \boldsymbol{\alpha}), F_{1}(\boldsymbol{u} ; \boldsymbol{\alpha}), F_{2}(\boldsymbol{u} ; \boldsymbol{\alpha}), F_{12}(\boldsymbol{u} ; \boldsymbol{\alpha})\right),
\end{aligned}
$$


where the $4 \times 4$-matrices $A$ and $C$ are given as follows

$$
A=\left(\begin{array}{cccc}
0 & -1 & 0 & 0 \\
1 & 0 & 0 & 0 \\
0 & 0 & 0 & 1 \\
0 & 0 & -1 & 0
\end{array}\right), \quad C=\left(\begin{array}{cccc}
1 & 0 & 0 & 0 \\
0 & 1 & 0 & 0 \\
0 & 0 & 1 & 0 \\
0 & 0 & 0 & -1 / \wp_{22}(\boldsymbol{\alpha})
\end{array}\right)
$$

The Schrödinger equation (4.11) and the hyperbolic equation (4.16) in this approach are, in this approach, the compatibility condition of the validity of the ansatz (3.25).

We shall show in the next sections how the Proposition 3.2 is used to derive 2D and 3D Schrödinger equations with a potential expressible in terms of Kleinian $\wp$-functions.

\section{The two Dimensional Schrödinger Equation With AN ABELIAN POTENTIAL}

Consider the Riemann surface of a curve $V(x, y)$ of genus 2 , in the form

$$
\begin{aligned}
w^{2} & =4 z^{5}+\lambda_{4} z^{4}+\lambda_{3} z^{3}+\lambda_{2} z^{2}+\lambda_{1} z+\lambda_{0} \\
& =4 \Pi_{k=1}^{5}\left(z-e_{k}\right)
\end{aligned}
$$

equipped with a homology basis $\left(\mathfrak{a}_{1}, \mathfrak{a}_{2} ; \mathfrak{b}_{1}, \mathfrak{b}_{2}\right) \in H_{1}(V, \mathbb{Z})$. The canonical holomorphic differentials and the associated meromorphic differentials of the second kind have the form

$$
\begin{aligned}
\mathrm{d} u_{1} & =\frac{\mathrm{d} z}{w}, \quad \mathrm{~d} u_{2}=\frac{z \mathrm{~d} z}{w}, \\
\mathrm{~d} r_{1} & =\frac{\lambda_{3} z+2 \lambda_{4} z^{2}+12 z^{3}}{4 w} \mathrm{~d} z, \quad \mathrm{~d} r_{2}=\frac{z^{2}}{w} \mathrm{~d} z .
\end{aligned}
$$

The fundamental Kleinian $\sigma$-function is expanded near $\boldsymbol{x}=0$ as follows

$$
\sigma\left(x_{1}, x_{2}\right)=x_{1}-\frac{1}{3} x_{2}^{3}+\frac{1}{24} \lambda_{2} x_{1}^{3}+o\left(\boldsymbol{x}^{3}\right) .
$$

Denote

$$
\begin{gathered}
\zeta_{i}(\boldsymbol{x})=\frac{\partial}{\partial x_{i}} \ln \sigma(\boldsymbol{x}), \quad i=1,2 \\
\wp_{i j}(\boldsymbol{x})=-\frac{\partial^{2}}{\partial x_{i} \partial x_{j}} \ln \sigma(\boldsymbol{x}), \quad i, j=1,2
\end{gathered}
$$

The equations of the Jacobi inversion problem

$$
x_{i}=\int_{\left(0, e_{1}\right)}^{\left(w_{1}, z_{1}\right)} \mathrm{d} u_{i}+\int_{\left(0, e_{2}\right)}^{\left(w_{2}, z_{2}\right)} \mathrm{d} u_{i}, \quad i=1,2
$$


are equivalent to an algebraic equation

$$
\mathcal{P}(z, \boldsymbol{x})=z^{2}-\wp_{22}(\boldsymbol{x}) z-\wp_{12}(\boldsymbol{x})=0,
$$

i.e., the pair $\left(z_{1}, z_{2}\right)$ is the pair of roots of (4.3). So we have

$$
\wp_{22}(\boldsymbol{x})=z_{1}+z_{2}, \wp_{12}(\boldsymbol{x})=-z_{1} z_{2} .
$$

The corresponding $w_{i}$ is expressed as

$$
w_{i}=\wp_{222}(\boldsymbol{x}) z_{i}+\wp_{122}(\boldsymbol{x}), \quad i=1,2 .
$$

The function $\wp_{11}(\boldsymbol{x})$ is expressed in terms of symmetric functions of the divisor as

$$
\wp_{11}(\boldsymbol{x})=\frac{F\left(z_{1}, z_{2}\right)-2 w_{1} w_{2}}{4\left(z_{1}-z_{2}\right)^{2}}
$$

where

$$
F\left(z_{1}, z_{2}\right)=\sum_{k=0}^{2} z_{1}^{k} z_{2}^{k}\left(2 \lambda_{2 k}+\lambda_{2 k+1}\left(z_{1}+z_{2}\right)\right) .
$$

The three functions $\wp_{11}, \wp_{12}, \wp_{22}$ are known to be algebraically dependent, being the coordinates of the quartic Kummer surface, which is given by the equation

$$
\operatorname{det}\left(\begin{array}{cccc}
\lambda_{0} & \frac{1}{2} \lambda_{1} & -2 \wp_{11} & -2 \wp_{12} \\
\frac{1}{2} \lambda_{1} & \lambda_{2}+4 \wp_{11} & \frac{1}{2} \lambda_{3}+2 \wp_{12} & -2 \wp_{22} \\
-2 \wp_{11} & \frac{1}{2} \lambda_{3}+2 \wp_{12} & \lambda_{4}+4 \wp_{22} & 2 \\
-2 \wp_{12} & -2 \wp_{22} & 2 & 0
\end{array}\right)=0
$$

where the variables $\wp_{22}=X, \wp_{12}=Y, \wp_{11}=Z$ are regarded as coordinates of the surface in $\mathbb{C}^{3}$.

We are now in a position to formulate the following theorem

Theorem 4.1. The following equality is valid for the six Baker functions $F_{0}(\boldsymbol{x} ; \alpha), F_{1}(\boldsymbol{x} ; \boldsymbol{\alpha}), F_{2}(\boldsymbol{x} ; \boldsymbol{\alpha}), F_{11}(\boldsymbol{x} ; \boldsymbol{\alpha}), F_{12}(\boldsymbol{x} ; \boldsymbol{\alpha})$ and $F_{22}(\boldsymbol{x} ; \boldsymbol{\alpha})$ in the case of genus two

$$
\begin{aligned}
& {\left[a F_{11}+b F_{12}+a \wp_{12}(\boldsymbol{\alpha}) F_{22}+\frac{1}{2} b \wp_{22}(\boldsymbol{\alpha}) F_{22}\right.} \\
& \left.\quad+\left(a \wp_{122}+\frac{1}{2} b \wp_{222}(\boldsymbol{\alpha})\right) F_{2}\right] \\
& \quad=\left[a\left(\wp_{11}(\boldsymbol{\alpha})-\wp_{12}(\boldsymbol{\alpha}) \wp_{22}(\boldsymbol{\alpha})+\frac{1}{4} \lambda_{2}\right)-\frac{1}{2} b \wp_{22}^{2}(\boldsymbol{\alpha})\right] F_{0}
\end{aligned}
$$

where $a, b$ are arbitrary and the $\lambda_{i}$ parameters of the curve (4.1). 
Proof. Consider the six functions,

$$
\sigma(\boldsymbol{x})^{2} F(\boldsymbol{x} ; \boldsymbol{\alpha}), \sigma(\boldsymbol{x})^{2} F_{i}(\boldsymbol{x} ; \boldsymbol{\alpha}), \quad \sigma(\boldsymbol{x})^{2} F_{i j}(\boldsymbol{x} ; \boldsymbol{\alpha}), i, j=1,2 .
$$

These are linearly dependent second order entire functions, satisfying (3.24) with $\gamma\left(\boldsymbol{m}, \boldsymbol{m}^{\prime}\right)=\boldsymbol{\zeta}^{T}(\boldsymbol{\alpha}) \boldsymbol{\Omega}\left(\boldsymbol{m}, \boldsymbol{m}^{\prime}\right)-\boldsymbol{E}^{T}\left(\boldsymbol{m}, \boldsymbol{m}^{\prime}\right) \boldsymbol{\alpha} \equiv \boldsymbol{k}(\boldsymbol{\alpha})$. Therefore there exist constants $c_{0}, c_{1}, c_{2}, c_{11}, c_{12}, c_{22} \neq 0$, such that

$$
c_{0} F_{0}(\boldsymbol{x} ; \boldsymbol{\alpha})+\sum_{k=1,2} c_{k} F_{k}(\boldsymbol{x} ; \boldsymbol{\alpha})+\sum_{i, j=1,2} c_{i j} F_{i j}(\boldsymbol{x} ; \boldsymbol{\alpha})=0
$$

Using the expansion (4.2) and the identity $\wp_{112}=\wp_{222} \wp_{12}-\wp_{122} \wp_{22}$, we arrive at the equations

$$
\begin{aligned}
c_{1} & =0, \\
c_{0}+c_{11} \wp_{11}(\boldsymbol{\alpha})+\frac{1}{4} c_{11} \lambda_{2}-c_{22} \wp_{22}(\boldsymbol{\alpha}) & =0, \\
2 c_{11} \wp_{12}(\boldsymbol{\alpha})+c_{12} \wp_{22}(\boldsymbol{\alpha})-2 c_{22} & =0, \\
-2 c_{2} \wp_{12}(\boldsymbol{\alpha})+c_{12} \wp_{122}(\boldsymbol{\alpha})+2 c_{22} \wp_{122}(\boldsymbol{\alpha}) & =0, \\
-c_{2} \wp_{22}(\boldsymbol{\alpha})-c_{11} \wp_{112}(\boldsymbol{\alpha})+c_{22} \wp_{222}(\boldsymbol{\alpha}) & =0, \\
2 c_{2}-2 c_{11} \wp_{122}(\boldsymbol{\alpha})-c_{12} \wp_{222}(\boldsymbol{\alpha}) & =0,
\end{aligned}
$$

whose solution reads

$$
\begin{aligned}
c_{11} & =a, \quad c_{12}=b, \\
c_{0} & =a\left(\wp_{11}(\boldsymbol{\alpha})-\wp_{12}(\boldsymbol{\alpha}) \wp_{22}(\boldsymbol{\alpha})+\frac{1}{4} \lambda_{2}\right)-\frac{1}{2} b \wp_{22}^{2}(\boldsymbol{\alpha}), \\
c_{22} & =a \wp_{12}(\boldsymbol{\alpha})+\frac{1}{2} b \wp_{22}(\boldsymbol{\alpha}), \\
c_{2} & =a \wp_{122}(\boldsymbol{\alpha})+\frac{1}{2} b \wp_{222}(\boldsymbol{\alpha}),
\end{aligned}
$$

where $a$ and $b$ are arbitrary. The equality (4.7) then follows immediately.

By choosing the parameters $a=1, b=0$ and then $a=0, b=1$ we get from the equality (4.7) the following equations on $\operatorname{Jac}(V) \times \operatorname{Jac}(V)$

$$
\begin{aligned}
& \left\{\left[\frac{\partial^{2}}{\partial x_{1}^{2}}-2 \wp_{11}(\boldsymbol{x})\right]+\wp_{12}(\boldsymbol{\alpha})\left[\frac{\partial^{2}}{\partial x_{2}^{2}}-2 \wp_{22}(\boldsymbol{x})\right]\right\} \Psi_{1}(\boldsymbol{x} ; \boldsymbol{\alpha}) \\
& =\frac{1}{4 \wp_{12}(\boldsymbol{\alpha})}\left(\lambda_{0}+\lambda_{2} \wp_{12}(\boldsymbol{\alpha})+\lambda_{4} \wp_{12}^{2}(\boldsymbol{\alpha})\right) \Psi_{1}(\boldsymbol{x} ; \boldsymbol{\alpha}),
\end{aligned}
$$

where

and

$$
\Psi_{1}(\boldsymbol{x} ; \boldsymbol{\alpha})=\Phi(\boldsymbol{x} ; \boldsymbol{\alpha}) \exp \left\{\frac{1}{2} \frac{\wp_{122}(\boldsymbol{\alpha})}{\wp_{12}(\boldsymbol{\alpha})} x_{2}\right\}
$$

$$
\wp_{22}(\boldsymbol{\alpha})\left\{\left[\frac{\partial^{2}}{\partial x_{1}^{2}}-2 \wp_{11}(\boldsymbol{x})\right]+2 \wp_{12}(\boldsymbol{\alpha})\left[\frac{\partial^{2}}{\partial x_{1} \partial x_{2}}-2 \wp_{12}(\boldsymbol{x})\right]\right\} \Psi_{2}(\boldsymbol{x} ; \boldsymbol{\alpha})
$$




$$
=-\wp_{22}^{2}(\boldsymbol{\alpha}) \Psi_{2}(\boldsymbol{x} ; \boldsymbol{\alpha}),
$$

where

$$
\Psi_{2}(\boldsymbol{x} ; \boldsymbol{\alpha})=\Phi(\boldsymbol{x} ; \boldsymbol{\alpha}) \exp \left\{-\frac{1}{2} \wp_{122}(\boldsymbol{\alpha}) x_{1}\right\} .
$$

Therefore the following theorems are valid for the case of genus two:

Theorem 4.2. Let $V$ be nonsingular hyperelliptic curve of genus 2 given by the equation (4.1). Let $\Phi_{B}(\boldsymbol{x} ; \boldsymbol{\alpha})$ be the standard Baker function on $\operatorname{Jac}(V) \times \operatorname{Jac}(V)$. Let $\boldsymbol{\Omega}=\left(\omega_{11}, \omega_{21}\right)^{T}, \boldsymbol{\Omega}^{\prime}=\left(\omega_{11}, \omega_{21}\right)^{T}$ be the real and imaginary half periods.

Then the following $2 D$ Schrödinger equation at a fixed energy level is valid

$$
\begin{array}{r}
\left\{\frac{\partial^{2}}{\partial x_{1}^{2}}+\frac{\partial^{2}}{\partial x_{2}^{2}}-\mathcal{U}_{1}(\boldsymbol{x})\right\} \Psi_{1}(\boldsymbol{x} ; \boldsymbol{\alpha}) \\
=\frac{1}{4}\left(\lambda_{0}+\lambda_{2}+\lambda_{4}\right) \Psi_{1}(\boldsymbol{x} ; \boldsymbol{\alpha}),
\end{array}
$$

where the smooth and real potential $\mathcal{U}_{1}(\boldsymbol{x})$ and wave function $\Psi_{1}(\boldsymbol{x} ; \boldsymbol{\alpha})$ have the form

$$
\begin{aligned}
\mathcal{U}_{1}(\boldsymbol{x}) & =2 \wp_{11}(\imath \boldsymbol{x}+\boldsymbol{\Omega})+2 \wp_{22}(\imath \boldsymbol{x}+\boldsymbol{\Omega}), \quad \boldsymbol{x} \in \mathbb{R}^{2}, \\
\Psi_{1}(\boldsymbol{x} ; \boldsymbol{\alpha}) & =\Phi_{B}\left(\imath \boldsymbol{x}+\boldsymbol{\Omega} ; \boldsymbol{\alpha}+\boldsymbol{\Omega}^{\prime}\right) \\
& \times \exp \left\{\frac{1}{2} \wp_{122}\left(\boldsymbol{\alpha}+\boldsymbol{\Omega}^{\prime}\right)\left(\imath x_{2}+\omega_{12}^{\prime}\right)\right\}, \quad \boldsymbol{x}, \boldsymbol{\alpha} \in \mathbb{R}^{2},
\end{aligned}
$$

and are restricted to the complex 1 -dimensional Bloch variety $\mathcal{B}_{1}$ given by the equation

$$
\mathcal{B}_{1}=\left\{(\boldsymbol{\alpha}) \mid \wp_{12}\left(\boldsymbol{\alpha}+\boldsymbol{\Omega}^{\prime}\right)=1\right\} .
$$

The vector of quasi-momentum is real and is given by the formula

$$
\boldsymbol{k}(\boldsymbol{\alpha})=\boldsymbol{\zeta}\left(\boldsymbol{\alpha}+\boldsymbol{\Omega}^{\prime}\right)-2 \varkappa\left(\boldsymbol{\alpha}+\boldsymbol{\Omega}^{\prime}\right)+\frac{1}{2} \wp_{122}\left(\boldsymbol{\alpha}+\boldsymbol{\Omega}^{\prime}\right)\left(\begin{array}{l}
0 \\
1
\end{array}\right) .
$$

Theorem 4.3. For the conditions of Theorem 4.2, the following hyperbolic equation at the zero energy level is valid

$$
\left\{\frac{\partial^{2}}{\partial x_{1} \partial x_{2}}-\mathcal{U}_{2}(\boldsymbol{x})\right\} \Psi_{2}(\boldsymbol{x} ; \boldsymbol{\alpha})=0,
$$

where the smooth and real potential $\mathcal{U}_{2}(\boldsymbol{x})$ and the wave function have the form

$$
\begin{aligned}
(4.18) \Psi_{2}(\boldsymbol{x} ; \boldsymbol{\alpha})= & \Phi_{B}\left(\imath \boldsymbol{x}+\boldsymbol{\Omega} ; \boldsymbol{\alpha}+\boldsymbol{\Omega}^{\prime}\right) \\
& \times \exp \left\{\frac{1}{2} \wp_{122}(\boldsymbol{\alpha})\left(\imath x_{1}+\omega_{21}^{\prime}\right)\right\}, \quad \boldsymbol{x}, \boldsymbol{\alpha} \in \mathbb{R}^{2},
\end{aligned}
$$


and is restricted to the complex 1-dimensional Bloch variety $\mathcal{B}_{2}$ given by the equation

$$
\mathcal{B}_{2}:\left\{(\boldsymbol{\alpha}) \wp_{22}\left(\boldsymbol{\alpha}+\boldsymbol{\Omega}^{\prime}\right)=0\right\} .
$$

The vector of quasi-momentum is real and given by the formula

$$
\boldsymbol{k}(\boldsymbol{\alpha})=\boldsymbol{\zeta}\left(\boldsymbol{\alpha}+\boldsymbol{\Omega}^{\prime}\right)-2 \varkappa\left(\boldsymbol{\alpha}+\boldsymbol{\Omega}^{\prime}\right)+\frac{1}{2} \wp_{222}\left(\boldsymbol{\alpha}+\boldsymbol{\Omega}^{\prime}\right)\left(\begin{array}{l}
1 \\
0
\end{array}\right) .
$$

The Bloch varieties $\mathcal{B}_{k} \subset \operatorname{Jac}(V), k=1,2$ are pull-backs of the varieties $\tilde{\mathcal{B}}_{k} \subset \operatorname{Kum}(V), k=1,2$ under the projection $\operatorname{Jac}(V) \rightarrow \operatorname{Kum}(V)$, where the $\tilde{\mathcal{B}}_{k}$ are given by the equations:

$$
\mathcal{B}_{1}=(X, Z): \operatorname{det}\left(\begin{array}{cccc}
\lambda_{0} & \frac{1}{2} \lambda_{1} & -2 Z & -2 \\
\frac{1}{2} \lambda_{1} & \lambda_{2}+4 Z & \frac{1}{2} \lambda_{3}+2 & -2 X \\
-2 Z & \frac{1}{2} \lambda_{3}+2 & \lambda_{4}+4 X & 2 \\
-2 & -2 X & 2 & 0
\end{array}\right)=0
$$

in the case of the 2D Schrödinger equation, and

$$
\mathcal{B}_{2}=(Y, Z): \operatorname{det}\left(\begin{array}{cccc}
\lambda_{0} & \frac{1}{2} \lambda_{1} & -2 Z & -2 Y \\
\frac{1}{2} \lambda_{1} & \lambda_{2}+4 Z & \frac{1}{2} \lambda_{3}+2 Y & 0 \\
-2 Z & \frac{1}{2} \lambda_{3}+2 Y & \lambda_{4} & 2 \\
-2 Y & 0 & 2 & 0
\end{array}\right)=0
$$

in the case of the hyperbolic equation.

The Bloch varieties $\mathcal{B}_{1,2}$ are algebraic curves, and it is straightforward to show that these curves are genus two hyperelliptic curves with a branching point at infinity. But in contrast with the case of genus one, these curves are not equivalent to the initial curve at generic $\lambda_{0}, \ldots, \lambda_{4}$.

We remark, that the explicit description of the varieties $\mathcal{B}_{k}, k=1,2$, which can be realized as algebraic curve of genus 4 with involution, and its link with the results of Veselov and Novikov [21, 20], is given in [12].

As an application of the above theory we show in Fig. 1 and Fig. 3 , the potential profiles as derived from (4.12), for two different sets of the parameter values (in Fig 2 and Fig 4 the corresponding level sets of these potentials are also shown). We see from these figures that the potentials are both real and smooth and have a spatial symmetry which is more general than the square or rectangular one considered in Ref. [10]. The full choice of more general crystal symmetries which can be found by properly appropriate choices of the parameters $\left\{e_{i}\right\}$, one can arrange is still under investigation. These properties make the above potentials suitable for physical applications. Similar results can be obtained for the potential (4.17) (for simplicity details are omitted). 


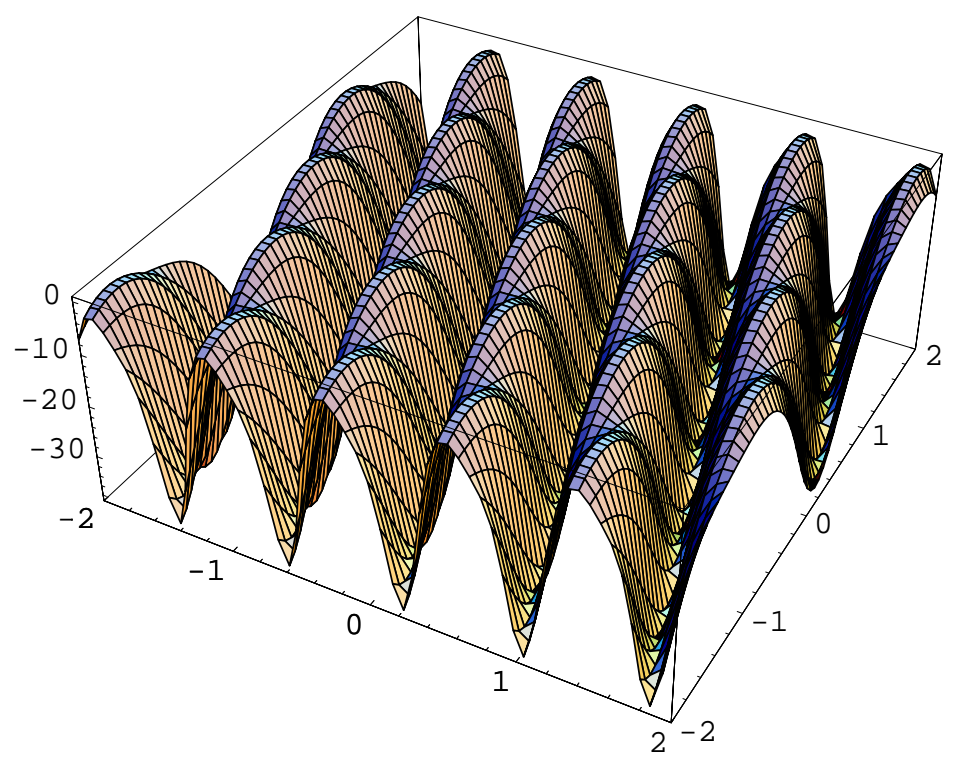

Figure 1. Potential profile as function of $x, y$ for the branching points $e_{1}=2 \sqrt{ } 3, e_{2}=3, e_{3}=0, e_{4}=-e_{2}, e_{5}=$ $-e_{1}$.

\section{The two Dimensional Schrödinger EQUATION With AN ELLIPTIC POTENTIAL}

In this section we shall construct an elliptic 2D Schrödinger equation with a potential which can be expressed in terms of an elliptic function by using the concept of elliptic solitons for the KdV equation. We shall show, that the real and nonsingular potential in the $(x, y)$ plane is provided by the dynamics over the locus of the Calogero-Moser system. The particle dynamics of the system over locus is essentially complex and was traditionally considered as non-physical. The equations for the Kleinian $\wp$-functions

$$
\begin{aligned}
& \wp_{2222}=6 \wp_{22}^{2}+4 \wp_{12}+\lambda_{4} \wp_{22}+\frac{1}{2} \lambda_{3}, \\
& \wp_{1222}=6 \wp_{22} \wp_{12}-2 \wp_{11}+\lambda_{4} \wp_{12},
\end{aligned}
$$

represent the KdV hierarchy for genus two curves with respect to the function

$$
\mathcal{U}=2 \wp_{22}(\boldsymbol{x})+\frac{1}{6} \lambda_{4}
$$




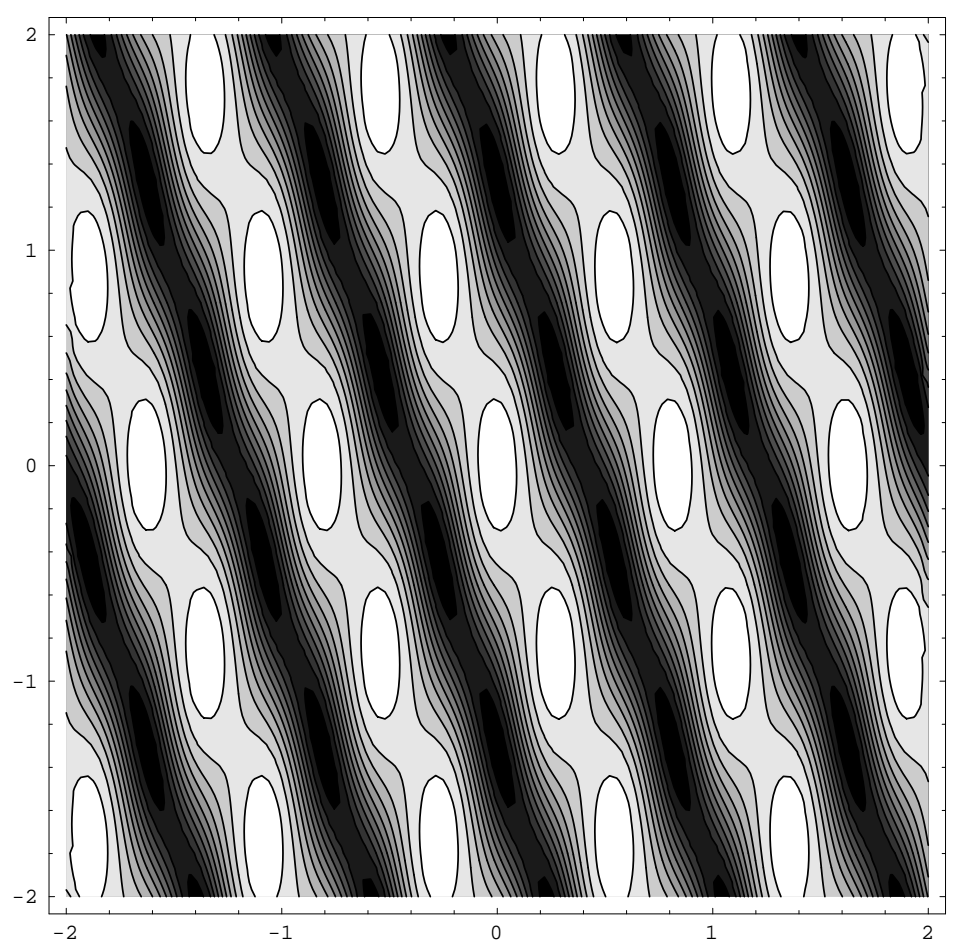

Figure 2. Topographic map of the potential in the case: $e_{1}=2 \sqrt{ } 3, e_{2}=3, e_{3}=0, e_{4}=-e_{2}, e_{5}=-e_{1}$. The regions between contours are shaded in such way that the ones with higher values are lighter.

The equation (5.1) becomes, after differentiation by $u_{2} \equiv x$, the $\mathrm{KdV}$ equation,

$$
\mathcal{U}_{x_{1}}=\frac{1}{2}\left(\mathcal{U}_{x_{2} x_{2} x_{2}}-6 \mathcal{U}_{x_{2}} \mathcal{u}\right)
$$

while the second equation (5.2) represents the second KdV flow which is stationary for the two-gap potential (5.3).

We further interpret the coordinates $\left(x_{1}, x_{2}\right)$ as space coordinates $(\boldsymbol{x})$ and set $\left(x_{1}, x_{2}\right)=(y, x)$. Consider the elliptic solution of the KdV equation

$$
\mathcal{U}(x, y)=2 \wp\left(x-f_{1}(y)\right)+2 \wp\left(x-f_{2}(y)\right)+2 \wp\left(x-f_{3}(y)\right),
$$

where $\wp(x)$ is the standard Weierstrass elliptic function, which represents the isospectral deformation of the two-gap Lamé potential $6 \wp(x)$ under the action of the KdV flow. The solution of the form (5.5) was introduced for the first time by Dubrovin and Novikov [16]; the general 


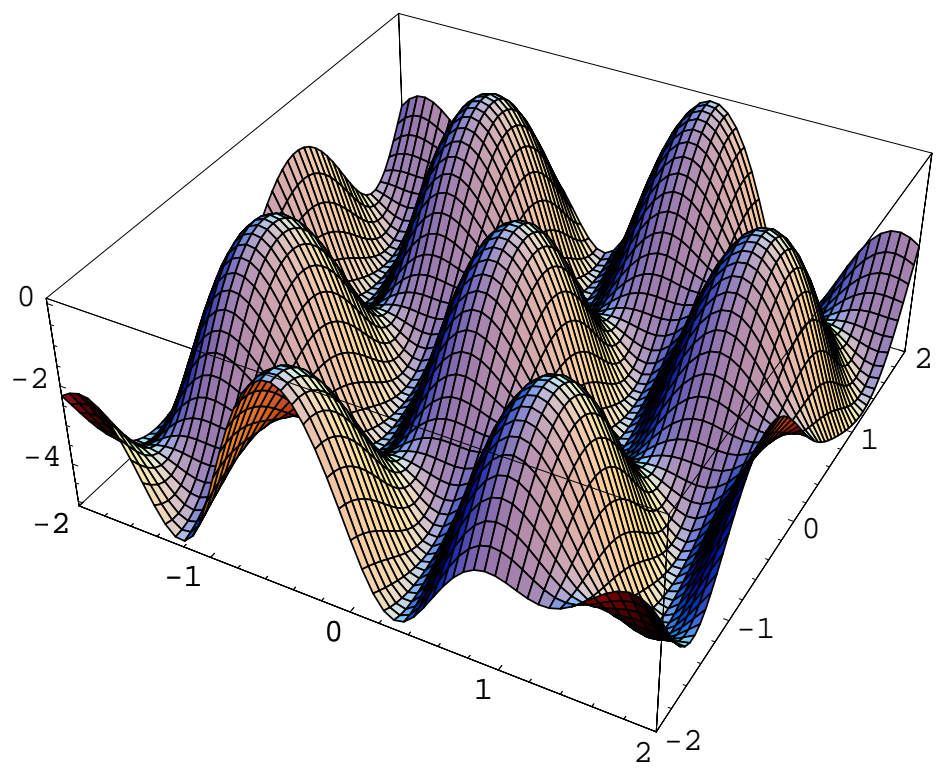

Figure 3. Same as in Fig. 1 for $e_{1}=2, e_{2}=1, e_{3}=$ $0, e_{4}=-1, e_{5}=-2$.

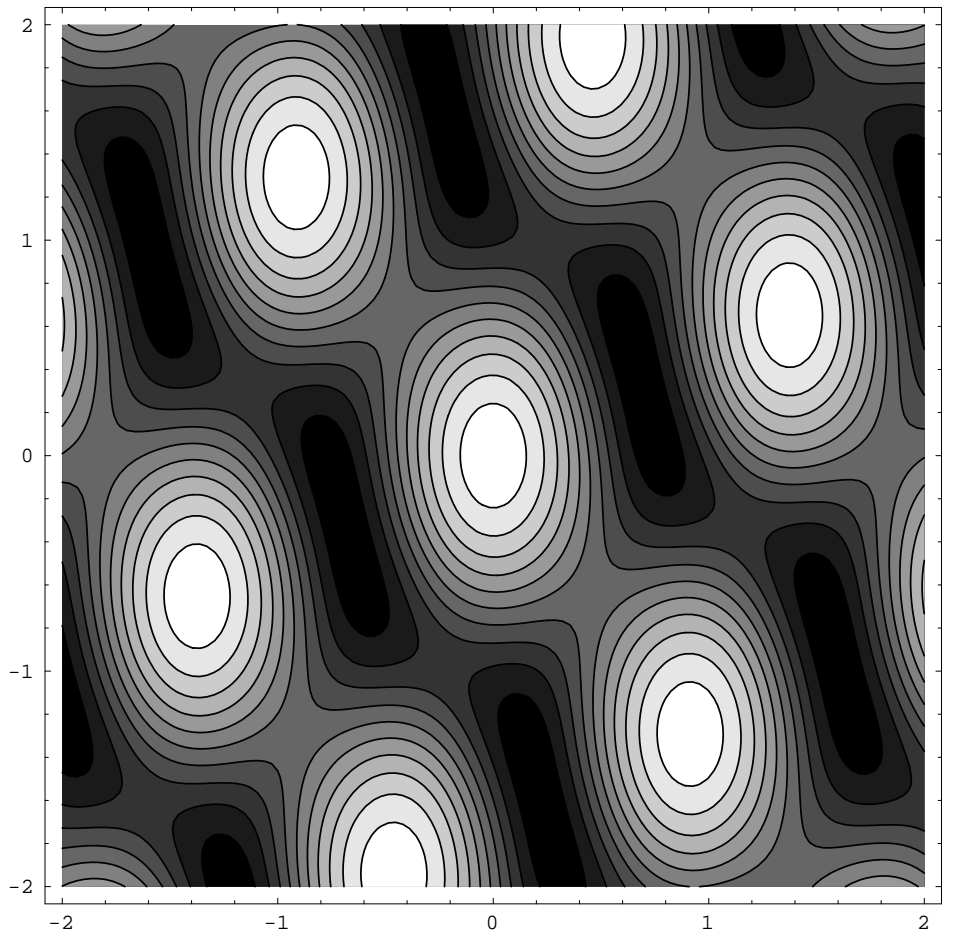

Figure 4. Same as in Fig. 2 for $e_{1}=2, e_{2}=1, e_{3}=$ $0, e_{4}=-1, e_{5}=-2$. 
case was investigated by Airault et al. [2]. We use this elliptic KdV solution below to construct a special solution of the 2D problem.

The ansatz (5.5) implies the following structure of the hyperelliptic, genus two, $\sigma$-function in the reduction case:

$$
\sigma(x, y)=\prod_{i=1}^{3} \sigma\left(x-f_{i}(y)\right),
$$

where $\sigma$ is the standard $\sigma$-function from the Weierstrass theory of elliptic functions. The associated algebraic curve is known to be of the form

$$
w^{2}=4\left(z^{2}-3 g_{2}\right)\left(z+3 e_{1}\right)\left(z+3 e_{2}\right)\left(z+3 e_{3}\right) .
$$

The remaining Kleinian $\wp$-functions, $\wp_{12}(\boldsymbol{x})$ and $\wp_{11}(\boldsymbol{x})$, are expressible from $(5.1,5.2)$ as differential polynomials of $\wp_{22}$, and have the form

$$
\begin{aligned}
\wp_{12}= & -3 \sum_{i<j=1,2,3} \wp\left(x-f_{i}(y)\right) \wp\left(x-f_{j}(y)\right)+\frac{9}{4} g_{2}, \\
\wp_{11}= & -27 \wp\left(x-f_{1}(y)\right) \wp\left(x-f_{2}(y)\right) \wp\left(x-f_{3}(y)\right) \\
& +3 \sum_{i<j=1,2,3} \wp^{\prime}\left(x-f_{i}(y)\right) \wp^{\prime}\left(x-f_{j}(y)\right) \\
& +\frac{21}{4} \sum_{i}^{3} \wp\left(x-f_{i}(y)\right),
\end{aligned}
$$

where $\wp$ is the standard Weierstrass $\wp$-function. This implies that the Abelian elliptic potential for the two-dimensional Schrödinger equation has the form

$$
\begin{aligned}
\mathcal{U}(x, y)= & -54 \wp\left(x-f_{1}(y)\right) \wp\left(x-f_{2}(y)\right) \wp\left(x-f_{3}(y)\right) \\
(5.10) & +6 \sum_{i<j=1,2,3} \wp^{\prime}\left(x-f_{i}(y)\right) \wp^{\prime}\left(x-f_{j}(y)\right)+\frac{25}{4} \sum_{i}^{3} \wp\left(x-f_{i}(y)\right) .
\end{aligned}
$$

For the proof we remark that the compatibility of the ansatz (5.5) with the KdV equation leads to the equations

$$
\begin{aligned}
& \frac{\mathrm{d} f_{1}}{\mathrm{~d} y}=-6 \mathfrak{P}_{12}-6 \mathfrak{P}_{13}, \\
& \frac{\mathrm{d} f_{2}}{\mathrm{~d} y}=-6 \mathfrak{P}_{21}-6 \mathfrak{P}_{23}, \\
& \frac{\mathrm{d} f_{3}}{\mathrm{~d} y}=-6 \mathfrak{P}_{31}-6 \mathfrak{P}_{33},
\end{aligned}
$$

with

(5.11) $\quad \mathfrak{P}_{12}^{\prime}+\mathfrak{P}_{13}^{\prime}=0, \quad \mathfrak{P}_{21}^{\prime}+\mathfrak{P}_{23}^{\prime}=0, \quad \mathfrak{P}_{31}^{\prime}+\mathfrak{P}_{33}^{\prime}=0$. 
Here we denote $\mathfrak{P}_{i j}=\wp\left(f_{i}(y)-f_{j}(y)\right)$. These equations represent the well known dynamics of the third flow of the integrable CalogeroMoser system, restricted to the stable points of the second flow. We remark, that the addition theorem for the Weierstrass $\wp$-function and the equations of the locus (5.11) allow us to rewrite the first group of the equations for $f_{i}$ in the form

$$
\frac{\mathrm{d} f_{1}}{\mathrm{~d} y}=6 \mathfrak{P}_{23}, \quad \frac{\mathrm{d} f_{2}}{\mathrm{~d} y}=6 \mathfrak{P}_{13}, \quad \frac{\mathrm{d} f_{3}}{\mathrm{~d} y}=6 \mathfrak{P}_{12}
$$

and therefore the elliptic KdV solution is given in the form

$$
\mathcal{U}(x, y)=2 \sum_{i<j=1,2,3} \wp\left(i x+\omega-6 \int_{0}^{y} \wp\left(f_{i}(y)-f_{j}(y)\right) \mathrm{d} y\right)
$$

with $\omega$ being a constant of integration. We shall find below the explicit expressions for the functions $\wp\left(f_{i}(y)-f_{j}(y)\right)$ and show that (5.13) represents an elliptic soliton, i.e. a real and smooth function which is doubly periodic in both $x$ and $y$. With this aim we consider the equations of the Jacobi inversion problem associated with the curve (5.7):

$$
\begin{aligned}
& \int_{(\infty, \infty)}^{\left(w_{1}, z_{1}\right)} \frac{\mathrm{d} z}{w}+\int_{(\infty, \infty)}^{\left(w_{2}, z_{2}\right)} \frac{\mathrm{d} z}{w}=x_{1} \equiv y, \\
& \int_{(\infty, \infty)}^{\left(w_{1}, z_{1}\right)} \frac{z \mathrm{~d} z}{w}+\int_{(\infty, \infty)}^{\left(w_{2}, z_{2}\right)} \frac{z \mathrm{~d} z}{w}=x_{2} \equiv x .
\end{aligned}
$$

The solution of the problem has the form

$$
\begin{aligned}
z_{1}+z_{2} & =2 \sum_{i=1}^{3} \wp\left(x-f_{i}(y)\right) \\
z_{1} z_{2} & =3 \sum_{i<j=1,2,3} \wp\left(x-f_{i}(y)\right) \wp\left(x-f_{j}(y)\right)-\frac{9}{4} g_{2},
\end{aligned}
$$

where we use $(5.1,5.2)$ and $(5.5)$ to derive $(5.16,5.17)$. Let us take the limit $x \rightarrow f_{1}(y)$. Then it follows from $(5.16,5.17)$

$$
z_{2} \rightarrow \infty, \quad z_{1} \rightarrow 3\left(\mathfrak{P}_{12}+\mathfrak{P}_{13}\right) \equiv-3 \mathfrak{P}_{23},
$$


and the equations of the Jacobi inversion problem will take the form

$$
\begin{aligned}
& \int_{\infty}^{-3 \mathfrak{P}_{23}} \frac{\mathrm{d} z}{2 \sqrt{\left(z^{2}-3 g_{2}\right)\left(z-3 e_{1}\right)\left(z-3 e_{2}\right)\left(z-3 e_{3}\right)}}=y \\
& \int_{\infty}^{-3 \mathfrak{P}_{23}} \frac{z \mathrm{~d} z}{2 \sqrt{\left(z^{2}-3 g_{2}\right)\left(z-3 e_{1}\right)\left(z-3 e_{2}\right)\left(z-3 e_{3}\right)}}=x .
\end{aligned}
$$

Two other pairs of equations of the form $(5.18,5.19)$ appears as the result of cyclic permutations of the indices $1,2,3$. To proceed we shall use the reduction formulae of Hermite, under which the hyperelliptic integrals in the l.h.s. of $(5.14,5.15)$ are reduced to elliptic integrals associated with the elliptic curves:

$$
\nu^{2}=4 \mu^{3}-g_{2} \mu-g_{3}, \quad \tilde{\nu}^{2}=4 \tilde{\mu}^{3}-\tilde{g}_{2} \tilde{\mu}-\tilde{g}_{3},
$$

whose moduli are linked by the relation

$$
\tilde{g}_{2}=\frac{4}{g_{2}^{2}}\left(3 g_{2}^{3}+27 g_{3}\right), \quad \tilde{g}_{3}=\frac{72}{g_{2}^{3}}\left(g_{3} g_{2}^{3}-3 g_{3}^{3}\right) .
$$

The equations for the cover are

$$
\begin{aligned}
(5.22)(\mu, \nu) & =\left(\frac{w}{27} \frac{z^{3}-9 g_{2}-54 g_{3}}{z^{2}-3 g_{2}}, \frac{z^{3}+27 g_{3}}{9\left(z^{2}-3 g_{2}\right)}\right), \\
(5.23)(\tilde{\nu}, \tilde{\mu}) & =\left(\sqrt{\left.\frac{2}{27 g_{2}^{3}} w\left(4 z^{2}-3 g_{2}\right), \frac{1}{3 g_{2}}\left(4 z^{3}-9 g_{2} z+9 g_{3}\right)\right) .} .\right.
\end{aligned}
$$

The reduction of the holomorphic differentials has the form

$$
\frac{\mathrm{d} z}{w}=\frac{2}{3 \sqrt{3 g_{2}}} \frac{\mathrm{d} \tilde{\nu}}{\tilde{\mu}} \quad \frac{z \mathrm{~d} z}{w}=\frac{1}{3} \frac{\mathrm{d} \mu}{\nu} .
$$

The application of the reduction formulae to equation (5.18) results in the following cubic equation with respect to $\mathfrak{P}_{23}$

$$
4 \mathfrak{P}_{23}^{3}-g_{2} \mathfrak{P}_{23}-\frac{1}{3} g_{3}+\frac{1}{9} g_{2} \tilde{\wp}\left(\frac{3}{2} \sqrt{3 g_{2}} y\right)=0 .
$$

Evidently the remaining two roots are exactly $\mathfrak{P}_{12}$ and $\mathfrak{P}_{13}$. Note that the equation (5.25) displays the following properties of the functions $\mathfrak{P}_{i j}$ on the locus:

$$
\begin{array}{r}
\mathfrak{P}_{12}+\mathfrak{P}_{13}+\mathfrak{P}_{23}=0 \\
\mathfrak{P}_{12} \mathfrak{P}_{13}+\mathfrak{P}_{23} \mathfrak{P}_{13}+\mathfrak{P}_{13} \mathfrak{P}_{23}=-\frac{1}{4} g_{2}
\end{array}
$$


Let us show that the application of the reduction formulae to the equation (5.19) leads to the equivalence. Indeed the substitution of the reduction formula (5.22) into (5.18) implies

$$
\wp\left(3 f_{1}\right)=\frac{\mathfrak{P}_{23}^{3}-g_{3}}{g_{2}-3 \mathfrak{P}_{23}^{2}} .
$$

We transform the left hand side:

$$
\begin{aligned}
\wp\left(3 f_{1}\right)= & \wp\left(\left\{f_{1}-f_{2}\right\}+\left\{f_{1}-f_{3}\right\}\right) \\
& \text { (because of the equality } \left.f_{1}+f_{2}+f_{3}=0\right) \\
= & -\mathfrak{P}_{12}-\mathfrak{P}_{13}+\frac{1}{4}\left[\frac{\mathfrak{P}_{12}^{\prime}-\mathfrak{P}_{13}^{\prime}}{\mathfrak{P}_{12}-\mathfrak{P}_{13}}\right]^{2}
\end{aligned}
$$

(because of the addition theorem for the Weierstrass elliptic function)

$$
=\mathfrak{P}_{23}+\frac{\mathfrak{P}_{23}^{\prime 2}}{\left(2 \mathfrak{P}_{12}+\mathfrak{P}_{23}\right)^{2}}
$$

(because (5.26) and locus equations, which imply

$$
\left.\mathfrak{P}_{23}^{\prime 2}-\mathfrak{P}_{12}^{\prime 2}=\left(\mathfrak{P}_{23}^{\prime}+\mathfrak{P}_{21}^{\prime}\right)\left(\mathfrak{P}_{23}^{\prime}+\mathfrak{P}_{12}^{\prime}\right)=0\right) \text {. }
$$

Further, the equation (5.27) leads to the relation

$$
\left(2 \mathfrak{P}_{12}+\mathfrak{P}_{23}\right)^{2}=g_{2}-3 \mathfrak{P}_{23}^{2} \text {. }
$$

Collecting all these equalities together we transform (5.28) to the equality

$$
\mathfrak{P}_{23}+\frac{\mathfrak{P}_{23}^{\prime 2}}{g_{2}-3 \mathfrak{P}_{23}^{2}} \equiv \frac{\mathfrak{P}_{23}^{3}-g_{3}}{g_{2}-3 \mathfrak{P}_{23}^{2}},
$$

whose validity can be checked directly.

Therefore we have proved the following proposition (a proof in compressed form was given in [9], see also [2], pg. 144)

Proposition 5.1. Let $(\mu, \nu)$ and $(\tilde{\nu}, \tilde{\mu})$ be two elliptic curves in the Weierstrass form with the moduli $g_{2}, g_{3}$ and $\tilde{g}_{2}, \tilde{g_{3}}$ given in (5.12). Denote by $\wp$ and $\tilde{\wp}$ the corresponding Weierstrass elliptic functions. Then the formula (5.13) describes the elliptic solution of the KdV equation with the integrands

$$
\wp\left(f_{i}(y)-f_{j}(y)\right)=\mathfrak{P}_{i, j}, \quad(i, j)=(1,2),(1,3),(2,3)
$$

being the roots of the cubic equation with coefficients depending on the moduli of the elliptic curve $g_{2}, g_{3}$ and the Weierstrass function $\tilde{\wp}$

$$
4 X^{3}-g_{2} X-\frac{1}{3} g_{3}+\frac{1}{9} g_{2} \tilde{\wp}\left(\frac{3}{2} \sqrt{3 g_{2}} y\right)=0 .
$$


We shall summarize the results as follows:

Theorem 5.2. Let $V$ be the Lamé curve

$$
w^{2}=4\left(z^{2}-3 g_{2}\right)\left(z+3 e_{1}\right)\left(z+3 e_{2}\right)\left(z+3 e_{3}\right),
$$

which covers 3-sheetedly the two elliptic curves

$$
\wp^{\prime 2}=4 \wp^{3}-g_{2} \wp-g_{3}, \quad{\tilde{\wp^{\prime}}}^{2}=4 \tilde{\wp}^{3}-\tilde{g}_{2} \tilde{\wp}-\tilde{g}_{3},
$$

whose moduli are linked by the relation

$$
\tilde{g}_{2}=\frac{4}{g_{2}^{2}}\left(3 g_{2}^{3}+27 g_{3}\right), \quad \tilde{g}_{3}=\frac{72}{g_{2}^{3}}\left(g_{3} g_{2}^{3}-3 g_{3}^{3}\right) .
$$

Then the wave function

$$
\Psi_{E}(\boldsymbol{x} ; \alpha, \beta)=\prod_{i=1}^{3} \Phi_{W}\left(i x+\omega-f_{i}(y) ; \alpha-f_{i}(\beta)\right),
$$

where the three functions $f_{i}$ are given by the formulae

$$
f_{i}(y)=-6 \int_{0}^{y} X_{i}(y) \mathrm{d} y,
$$

and $X_{i}$ are the three roots of the cubic equation

$$
4 X^{3}-g_{2} X-\frac{1}{3} g_{3}+\frac{1}{9} g_{2} \tilde{\wp}\left(\frac{3}{2} \sqrt{3 g_{2}} y\right)=0
$$

satisfy the 2D Schrödinger equation

$$
\left\{\frac{\partial^{2}}{\partial x^{2}}+\frac{\partial^{2}}{\partial y^{2}}-\mathcal{U}(x, y)\right\} \Psi_{E}=\Lambda \Psi_{E}
$$

with the elliptic smooth and nonseparable potential given by the formula $\mathcal{U}(x, y)=-54 \wp\left(x-f_{1}(y)\right) \wp\left(x-f_{2}(y)\right) \wp\left(x-f_{3}(y)\right)$

$$
+6 \sum_{i<j=1}^{3} \wp^{\prime}\left(x-f_{i}(y)\right) \wp^{\prime}\left(x-f_{j}(y)\right)+\frac{25}{4} \sum_{i}^{3} \wp\left(x-f_{i}(y)\right),
$$

on the fixed energy level $\Lambda=-36 g_{2} g_{3}+36 g_{3}$. The spectral variety is a one dimensional variety given by the equation

$$
3 \sum_{i<j=1,2,3} \wp\left(\alpha-f_{i}(\beta)\right) \wp\left(\alpha-f_{j}(\beta)\right)-\frac{9}{4} g_{2}=1 .
$$

We remark that the Bloch variety (5.35), which is given as a hyperelliptic curve, is uniformizable in this case by elliptic functions with moduli (5.31).

We note that we could consider other two-gap elliptic potentials in an analogous fashion. 


\section{The 3D SchröDinger EQUATION}

We consider the case of the curve defined by the equation

$$
y^{2}=4 x^{7}+\sum_{0}^{6} \lambda_{j} x^{j} .
$$

The principal matrix $H$ is given by (3.10) and is a $5 \times 5$ matrix of the form

$$
H=\left(\begin{array}{ccccc}
\lambda_{0} & \frac{1}{2} \lambda_{1} & -2 \wp_{11} & -2 \wp_{12} & -2 \wp_{13} \\
\frac{1}{2} \lambda_{1} & 4 \wp_{11}+\lambda_{2} & 2 \wp_{12}+\frac{1}{2} \lambda_{3} & 4 \wp_{13}-2 \wp_{22} & -2 \wp_{23} \\
-2 \wp_{11} & 2 \wp_{12}+\frac{1}{2} \lambda_{3} & 4 \wp_{22}-4 \wp_{13}+\lambda_{4} & 2 \wp_{23}+\frac{1}{2} \lambda_{5} & -2 \wp_{33} \\
-2 \wp_{12} & 4 \wp_{13}-2 \wp_{22} & 2 \wp_{23}+\frac{1}{2} \lambda_{5} & 4 \wp_{33}+\lambda_{6} & 2 \\
-2 \wp_{13} & -2 \wp_{23} & -2 \wp_{33} & 2 & 0
\end{array}\right) .
$$

The expansion of the $\sigma$-function of genus 3 in the vicinity of $\boldsymbol{u}=0$ (found for the first time in [14]) has the form

$$
\begin{aligned}
\sigma\left(u_{1}, u_{2}, u_{3}\right)= & u_{1} u_{3}-u_{2}^{2} \\
& -\frac{1}{24}\left(2 \lambda_{0} u_{1}^{4}+2 \lambda_{1} u_{1}^{3} u_{2}+\lambda_{2} u_{1}^{2}\left(3 u_{2}^{2}-u_{1} u_{3}\right)+8 u_{2} u_{3}^{3}\right. \\
& \left.+2 \lambda_{3} u_{1} u_{2}^{3}+2 \lambda_{4} u_{2}^{4}+2 \lambda_{5} u_{2}^{3} u_{3}+\lambda_{6} u_{3}^{2}\left(3 u_{2}^{2}-u_{1} u_{3}\right)\right) \\
& + \text { higher order terms. }
\end{aligned}
$$

We are in position now to prove the following theorem

Theorem 6.1. Let $F_{i, j}(\boldsymbol{x} ; \boldsymbol{\alpha})$ be the functions (3.19). Then the following equalities are valid for all $\boldsymbol{x} ; \boldsymbol{\alpha} \in \mathbb{C}^{3}$

$$
\begin{aligned}
& \quad 4\left(\wp_{113}(\boldsymbol{\alpha})-\wp_{122}(\boldsymbol{\alpha})\right) F_{33}(\boldsymbol{x} ; \boldsymbol{\alpha})-4\left(2 F_{13}(\boldsymbol{x} ; \boldsymbol{\alpha})+F_{22}(\boldsymbol{x} ; \boldsymbol{\alpha})\right) \wp_{133}(\boldsymbol{\alpha}) \\
& \quad+8 F_{23}(\boldsymbol{x} ; \boldsymbol{\alpha}) \wp_{123}(\boldsymbol{\alpha})+4 F_{11}(\boldsymbol{x} ; \boldsymbol{\alpha}) \wp_{333}(\boldsymbol{\alpha})= \\
& (6.2) \Lambda F_{0}(\boldsymbol{x} ; \boldsymbol{\alpha}),
\end{aligned}
$$

where

$$
\begin{aligned}
\Lambda= & 4\left(2 \wp_{13}(\boldsymbol{\alpha})-\wp_{22}(\boldsymbol{\alpha})\right) \wp_{133}(\boldsymbol{\alpha})+\lambda_{6 \wp_{122}}(\boldsymbol{\alpha}) \\
& -4 \wp_{33}(\boldsymbol{\alpha}) \wp_{113}(\boldsymbol{\alpha})-\lambda_{2} \wp_{333}(\boldsymbol{\alpha})+4 \wp_{33}(\boldsymbol{\alpha}) \wp_{122}(\boldsymbol{\alpha}) \\
& -4 \wp_{11}(\boldsymbol{\alpha}) \wp_{333}(\boldsymbol{\alpha})-\lambda_{6} \wp_{113}(\boldsymbol{\alpha}),
\end{aligned}
$$

and

$$
\begin{aligned}
4\left(2 \wp_{123}(\boldsymbol{\alpha})-\wp_{222}(\boldsymbol{\alpha})\right) F_{33}(\boldsymbol{x} ; \boldsymbol{\alpha})+8\left(\wp_{223}(\boldsymbol{\alpha})-\wp_{133}(\boldsymbol{\alpha})\right) F_{23}(\boldsymbol{x} ; \boldsymbol{\alpha}) \\
-4\left(2 F_{13}(\boldsymbol{x} ; \boldsymbol{\alpha})+F_{22}(\boldsymbol{x} ; \boldsymbol{\alpha})\right) \wp_{233}(\boldsymbol{\alpha})+8 F_{12}(\boldsymbol{x} ; \boldsymbol{\alpha}) \wp_{3,3,3}(\boldsymbol{\alpha})= \\
\quad \Lambda F_{0}(\boldsymbol{x} ; \boldsymbol{\alpha}),
\end{aligned}
$$

where

$\Lambda=4\left(2 \wp_{13}-\wp_{22}\right) \wp_{233}-2 \lambda_{6} \wp_{123}+\lambda_{6} \wp_{222}-8 \wp_{33} \wp_{123}+4 \wp_{33} \wp_{222}$. 
Proof. Let us take (3.20) in the case $g=3$ and assume $c_{13}=2 c_{22}$. Then we have

$$
c_{0} F_{0}+\sum_{i=1}^{3} c_{i} F_{i}+\sum_{i \leq j=1, \ldots 3} C_{i+j} F_{i, j}=0,
$$

where we denote $c_{i j}=C_{i+j}$. Let us multiply (6.3) by $\sigma^{2}(\boldsymbol{\alpha})$ and expand the resulting equality in a power series in $u_{1}, u_{2}, u_{3}$ by using (6.1). We find

$$
c_{1}=c_{2}=c_{3}=0,
$$

and 6 equations to define $C_{0}, C_{2}, C_{3}, C_{4}, C_{5}, C_{6}$ :

$$
\begin{aligned}
-2 C_{5}+2 C_{4} \wp_{33}+2 C_{2} \wp_{13}+2 C_{3} \wp_{23} & =0, \\
-4 C_{2} \wp_{12}+\left(-4 \wp_{22}+8 \wp_{13}\right) C_{3} & \\
+\left(\lambda_{5}+4 \wp_{23}\right) C_{4}+\left(8 \wp_{33}+2 \lambda_{6}\right) C_{5}+4 C_{6} & =0, \\
\left(\lambda_{2}-4 \wp_{11}\right) C_{2}+\left(2 \lambda_{3}+8 \wp_{12}\right) C_{3}+\left(4 \lambda_{4}+12 \wp_{22}-8 \wp_{13}\right) C_{4} & \\
+\left(2 \lambda_{5}+8 \wp_{23}\right) C_{5}+\left(\lambda_{6}-4 \wp_{33}\right) C_{6}+4 C_{0} & =0, \\
\left(\lambda_{2}+4 \wp_{11}\right) C_{2}+\left(8 \wp_{13}-4 \wp_{22}\right) C_{4}+\left(\lambda_{6}+4 \wp_{33}\right) C_{6}+4 C_{0} & =0, \\
\lambda_{1} C_{2}+\left(8 \wp_{11}+2 \lambda_{2}\right) C_{3} & \\
+\left(\lambda_{3}+4 \wp_{12}\right) C_{4}+\left(8 \wp_{13}-4 \wp_{22}\right) C_{5}-4 C_{6} \wp_{23} & =0 \\
4 \lambda_{0} C_{2}+2 \lambda_{1} C_{3}-8 C_{5} \wp_{12}-8 C_{6} \wp_{13}-8 C_{4} \wp_{11} & =0 .
\end{aligned}
$$

We find from the third equation

$$
C_{0}=-\left(\wp_{11}-\frac{1}{4} \lambda_{2}\right) C_{2}+\left(\wp_{22}-2 \wp_{13}\right) C_{4}-\left(\wp_{33}+\frac{1}{4} \lambda-6\right) C_{6} .
$$

To define the remaining parameters, add the third equation to the fourth and consider the remaining 5 equations. They are homogeneous equations with respect to the 5 variables $C_{2}, C_{3}, C_{4}, C_{5}, C_{6}$, whose matrix is exactly the matrix $H$. Because the matrix $H$ has rank 3 , the general solution depends on two arbitrary variables $a=C_{2}$ and $b=C_{3}$. The remaining variables are computed by applying the formula (3.15).

The matrix of the first equation (6.2) is

$$
M_{1}=\left[\begin{array}{ccc}
\wp_{333} & 0 & -\wp_{133} \\
0 & -\wp_{133} & \wp_{123} \\
-\wp_{133} & \wp_{123} & \wp_{113}-\wp_{122}
\end{array}\right]
$$


and the matrix of the second equation (6.3) is

$$
M_{2}=\left[\begin{array}{ccc}
0 & \wp_{333} & -\wp_{233} \\
\wp_{333} & -\wp_{233} & -\wp_{133}+\wp_{223} \\
-\wp_{233} & -\wp_{133}+\wp_{223} & 2 \wp_{123}-\wp_{222}
\end{array}\right]
$$

It can be checked by direct substitution that in the rational limit

$$
\sigma\left(\alpha_{1}, \alpha_{2}, \alpha_{3}\right)=-\alpha_{2}^{2}+\alpha_{1} \alpha_{3}-\frac{1}{3} \alpha_{2} \alpha_{3}^{3}+\frac{1}{45} \alpha_{3}^{6}
$$

there are regions in $\left(\alpha_{1}, \alpha_{2}, \alpha_{3}\right)$ space where the principal minors are all nonpositive.

\section{ACKNowledGements}

MS wishes to acknowledge support from INFM, the Edinburgh Mathematical Society, and by the Royal Society of Edinburgh. JCE, VZE and MS also acknowledge support under EPSRC grant GR/R23336/10, the LOCNET EU network HPRN-CT-1999-00163. JCE and VZE are grateful for the support of the Isaac Newton Institute during the Integrable Systems programme where the final draft of this paper was prepared.

\section{REFERENCES}

[1] M. Abramowitz and I. A. Stegun. Handbook of mathematical functions. Dover, 1965.

[2] H. Airault, H. P. McKean, and J. Moser. Rational and elliptic solutions of the $\mathrm{KdV}$ equation and a related many-body problem. Comm. Pure and Appl. Math., 30:94-148, 1977.

[3] H. F. Baker. Abels theorem and the allied theory including the theory of theta functions. Cambridge Univ. Press, Cambridge, 1897, reprinted 1995.

[4] H. F. Baker. Multiply Periodic Functions. Cambridge Univ. Press, Cambridge, 1907.

[5] V. G. Bar'yakhtar, E. D. Belokolos, and A. M. Korostil. Superconductivity of layered systems in a finite-gap potential model. Metalofizika, 13(5):3-8, 1991.

[6] V. G. Bar'yakhtar, E. D. Belokolos, and A. M. Korostil. A new method for calculating the electron spectrum in solids. application to high-temperature superconductors. Phys. Stat. Sol. (b), 169(1):105-114, 1992.

[7] H. Bateman and A. Erdelyi. Higher Transcendental Functions, volume 2. McGraw-Hill, New York, 1955.

[8] E. D. Belokolos. Peierls-Fröhlich problem and potentials with finite number of gaps I. Teoret. Mat. Fiz., 45:268-275, 1980.

[9] E. D. Belokolos, A. I. Bobenko, V. Z. Enolskii, A. R. Its, and V. B. Matveev. Algebro Geometrical Aproach to Nonlinear Integrable Equations. Springer, Berlin, 1994. 
[10] E. D. Belokolos, J. C. Eilbeck, V. Z. Enolskii, and M. Salerno. Exact energy bands and Fermi surfaces of separable Abelian potentials. J. Phys. A: Math. Gen., 34:943-959, 2001.

[11] V. M. Buchstaber and V. Z. Enolskii. Abelian Bloch solutions of two dimensional Schrödinger equation. Russian Maths. Surveys, 50(1):195-197, 1995.

[12] V. M. Buchstaber, V. Z. Enolskii, and D. V. Leykin. Bloch variety of 2D Schrödinger equation with Abelian potential. In preparation.

[13] V. M. Buchstaber, V. Z. Enolskii, and D. V. Leykin. Hyperelliptic Kleinian functions and applications. In V. M. Buchstaber and S. P. Novikov, editors, Solitons, Geometry and Topology: On the Crossroad, pages 1-34. Advances in Math. Sciences, AMS Translations, Series 2, Vol. 179, Moscow State University and University of Maryland, College Park, 1997.

[14] V. M. Buchstaber, V. Z. Enolskii, and D. V. Leykin. Kleinian functions, hyperelliptic Jacobians and applications. In S. P. Novikov and I. M. Krichever, editors, Reviews in Mathematics and Mathematical Physics, volume 10:2, pages 1-125, London, 1997. Gordon and Breach.

[15] B. A. Dubrovin, I. M. Krichever, and S. P. Novikov. The Schrödinger equation in a periodic field on Riemann surface. Dokl. AN SSR, 229(1):15-18, 1976.

[16] B. A. Dubrovin and S. P. Novikov. Periodic and quasiperiodic analogues of multisoliton solutions of Korteweg-de Vries equation. Soviet JETP, 67(6):21312143, 1974.

[17] J. C. Eilbeck and V. Z. Enolskii. Bilinear operators and the power series for the Weierstrass $\sigma$-function. J. Phys. A: Math. Gen., 33:791-794, 2000.

[18] I. M. Krichever. The method of algebraic geometry in the theory of nonlinear equations. Russian. Math. Surveys, 32:180-208, 1977.

[19] S. P. Novikov. Two dimensional Schrödinger operators in periodic fields. Itogi Nauki i Techniki, VINITI, 23:3-35, 1983.

[20] A. P. Veselov and S. P. Novikov. Finite zone two dimensional periodic Schrödinger operators: case of potential. Dokl. AN. SSSR, 279(4):784-788, 1984.

[21] A. P. Veselov and S. P. Novikov. Finite zone two dimensional periodic Schrödinger operators: explicit formulae and evolution equations. Dokl. AN. SSSR, 279(1):20-24, 1984.

[22] K. Weierstrass. Zur Theorie der elliptischen Funktionen. In Mathematische werke von Karl Weierstrass; herausgegeben unter Mitwirkung einer von der Königlich preussischen Akademie der Wissenschaften eingesetzten Commission, volume 2, pages 245-255, Berlin, 1894. Originally published in Sitzungsberichte der Akademie der Wissenshaften zu Berlin, 443-451, 1882.

V. M. Buchstaber: Steklov Mathematical Institute, Russian AcadEmy of Sciences, Gubkina str. 8 Moscow, GSP-1, 117966, Russia

E-mail address: buchstab@mendeleevo.ru, buchstab@mi.ras.ru

J. C. Eilbeck: Department of Mathematics, Heriot-Watt University, EDINBURGH EH14 4AS, UK

E-mail address: J.C.Eilbeck@ma.hw.ac.uk 
V. Z. Enolskit: Theoretical Physics Division, National Academy of Sciences of Ukraine, Institute of Magnetism, 36-B Vernadsky str., KIEV-680, 252142, UKRAINE

E-mail address: vze@imag.kiev.ua, vze@ma.hw.ac.uk

D. V. Leykin: Theoretical Physics Division, NASU Institute of MagNetism, 36-B Vernadsky STR., Kiev-680, 252142, UkRaine

E-mail address: dile@imag.kiev.ua

M. Salerno: Dipartimento di Scienze Fisiche "E. R. Caianiello" and infM Unitá di Salerno, via S.Allende, I-84081, Baronissi (SA), Italy

E-mail address: salerno@sa.infn.it 